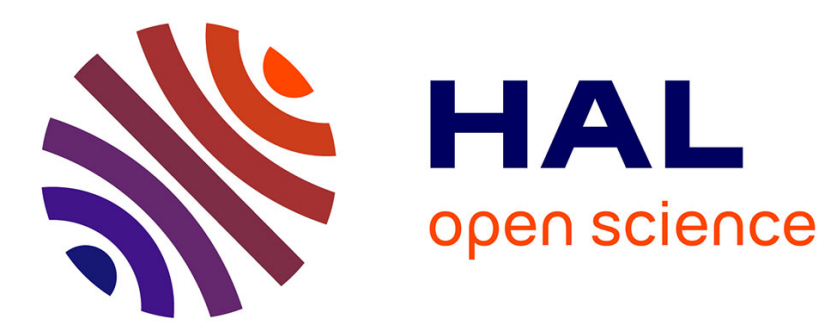

\title{
Le tonneau en Gaule romaine Élise Marlière
}

\section{To cite this version:}

Élise Marlière. Le tonneau en Gaule romaine. Gallia - Archéologie de la France antique, 2001, La viticulture en Gaule, 58, pp.181-201. 10.3406/galia.2001.3179 . hal-01909421

\section{HAL Id: hal-01909421 \\ https://hal.science/hal-01909421}

Submitted on 20 Jan 2020

HAL is a multi-disciplinary open access archive for the deposit and dissemination of scientific research documents, whether they are published or not. The documents may come from teaching and research institutions in France or abroad, or from public or private research centers.
L'archive ouverte pluridisciplinaire HAL, est destinée au dépôt et à la diffusion de documents scientifiques de niveau recherche, publiés ou non, émanant des établissements d'enseignement et de recherche français ou étrangers, des laboratoires publics ou privés.

\section{(이)(\$)}

Distributed under a Creative Commons Attribution - NonCommercial - NoDerivatives| 4.0 


\title{
LE TONNEAU EN GAULE ROMAINE
}

\author{
Élise MARLIÈRE
}

\begin{abstract}
Mots-clés. Tonneau, barrique, tonnelel, tonnelier, viticulture, vin, bière, salaisons de poisson, amphore, camp militaire, puits, commerce, transport.

Key-words. Cask, barrel, keg, cooper, vine growing, wine, beer, salt-fish, amphora, military camp, well, trade, transport.

Résumé. Crâce à l'étude de plus de 200 fûts découverts en Gaule, en Bretagne et sur le limes rhénano-danubien, il a été possible de tracer des carles de leur diffusion du $I^{e r}$ s. avant notre ère au $I V^{p}$ s. de notre ère, et d'établir une première typologie du tonneau, dont trois groupes sur six sont destinés aux camps militaires. De l'époque augustéenne jusqu'à la fin du Pro s. de notre ère, la moyenne vallée du Rhône apparaît comme une très importante zone de production qu'il faut mettre en relation avec l'approvisionnement des troupes stationnées sur le limes. D'autres centres de production ont été distingués au cour de régions viticoles : dans le Bordelais, autour de l'estuaire de la Loire, en Bourgogne et dans le pays mosellan.
\end{abstract}

\begin{abstract}
Thanks to the study of more than 200 casks discovered in Britain, in Gaul and on the rhenano-danubian limes, it is possible to draw maps of distribution from the $1^{\text {st }}$ century $B C$ to the $4^{\text {th }}$ century $A D$ and to propose the first barrel typology. Among the six groups defined three are for the military market. From the Augustan period to the end of the $1^{\text {st }}$ century AD, the middle Rhone valley appears to be one of the main casks production areas to connect with the military supply of the limes. Some other barrel siles of production have been characterized in the heart of vine growing regions : around Bordeaux and Nantes, in Burgundy and in the Moselle valley.
\end{abstract}

Le transport de certaines denrées dans les provinces occidentales de l'Empire romain n'a été envisagé pendant longtemps qu'au travers des emballages céramiques, dont la masse des vestiges archéologiques occultait l'existence d'une réelle alternative à l'amphore, en l'occurrence le tonneau. Cependant, à la lumière des travaux réalisés ces dernières années, il a semblé possible de réexaminer la place que ce dernier occupa dans le commerce antique. En effet, de nombreux fîts ont pu être inventoriés, dont une grande majorité provient de puits dans lesquels ils avaient été réemployés comme cuvelage. Cette utilisation secondaire a permis à ces fragiles témoins de résister au temps, puisqu'un milieu humide et anaérobie est indispensable à la conservation du bois. Mais bien que cela soit une grande chance pour l'étude de ces conteneurs, les conditions nécessairement spécifiques à leur réutilisation posent différents problèmes liés à la répartition, à la chronologie et à la typologie, et nous offrent une vision passablement déformée de la réalité. L'apport des textes et de l'iconographie ainsi que les indices laissés par les tonneliers (outils et monuments funéraires) permettent toutefois de rétablir une image plus fiable de l'usage du tonneau dans le monde romain occidental, et plus particulièrement en Gaule ${ }^{212}$.

\section{I.FS VFSTIGES DE TONNEAUX}

La réalisation d'un catalogue a permis de recenser 202 tonneaux qui se répartissent presque exclusivement en Gaule, en Bretagne et dans les camps militaires du limes rhénano-danubien (fig. 101 et tabl. V, p. 194-201).

212. Cette participation au présent dossier consacré à la viticulture en Gaule est tirée d'une thèse de doctorat intitulée Amphores, tonneaux et outres. Contribution à l'histoire économique de la Gaule Belgique occidentale, codirigée par Fanette Laubenheimer et Arthur Muller, et soutenue à l'université Charles-de-Gaulle-Lille 3 en janvier 2000. 


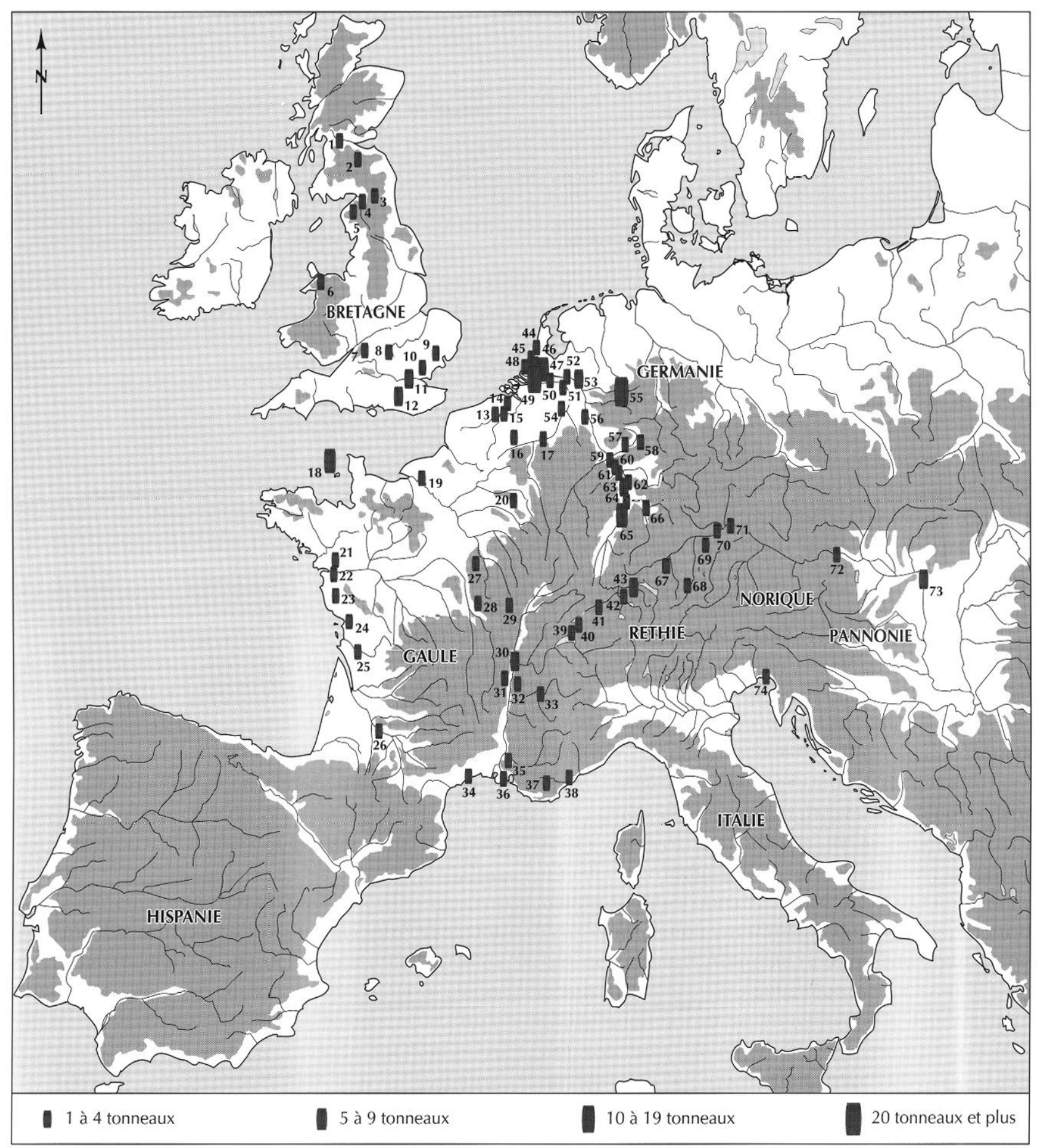

Fig. 101 - Carte de répartition des découverles de lonneaux antiques (fond de carte (C) M. leugère, M. P), CNRS, 1994).

BNE:TAGNE : 1, Bar Hill ; 2, Netustead ; 3, Vindolanda ; 4, Carlisle; 5, Kirkby Thore ; 6, Segontium ; 7, Haverfield ; 8, Stone ; 9, Colchester; 10, Wickford; 11, Londres; 12 , Silchester.

GAltL: : 13, Kuurne; 14, Aardenburg; 15, Harelbeke; 16, Bavay; 17, Erden; 18, Guernesey ; 19, Rouen ; 20, Reims; 21, Blain; 22, Rezé ; 23, Le Bernard ; 24, Mortantambe ; 25, Sainles ; 26, Lectoure ; 27, La Chapelle-Vaupelleigne ; 28, Champallement ; 29, Aulun ; 30, Lyon ; 31, Saint-Romain-en-Gal ; 32, Vienne ; 33, Grenoble; 34, Lattes ; 35, Glanum ; 36, Fos-sur-Mer ; 37, Pignans ; 38, Irójus ; 39, Nyon ; 40, Avenches ; 41, Kaiseraugst ; 42, Windisch ; 43, Oberwinterthur.

L.Imes rHLNAN : 44, Velsen ; 45, Katun̈k-sur-Mer; 46, Valkenbourg; 47, Arentsburg-Voorburg; 48, Rijswijk; 49, Vechlen ; 50, Druten ; 51, Woerd de Ressen ; 52, Nimègue ; 53, Xanten ; 54, Mülheim ; 55, Oberaden ; 56, Neuss ; 57, Saalburg ; 58, Okarben ; 59, Mayence ; 60, (ross Gerau; 61, Worms; 62, Viernheim; 63, Rheingonheim ; 64, Rheinzabern; 65, Strasbourg; 66, Ohrigen.

Limis isnubikN : 67, Risstissen ; 68, Kempten ; 69, Augsburg; 70, Manching ; 71, Regensburg ; 72, Carnuntum ; 73, Budapesl. ITAI.IL: : 74, Grado. 


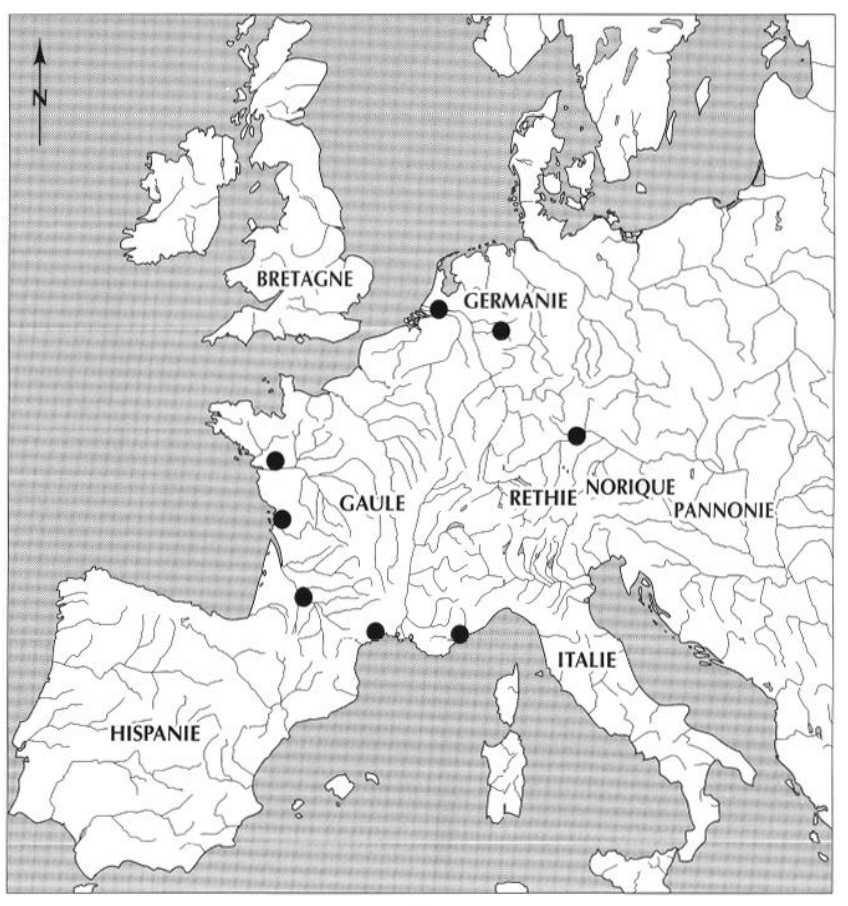

1

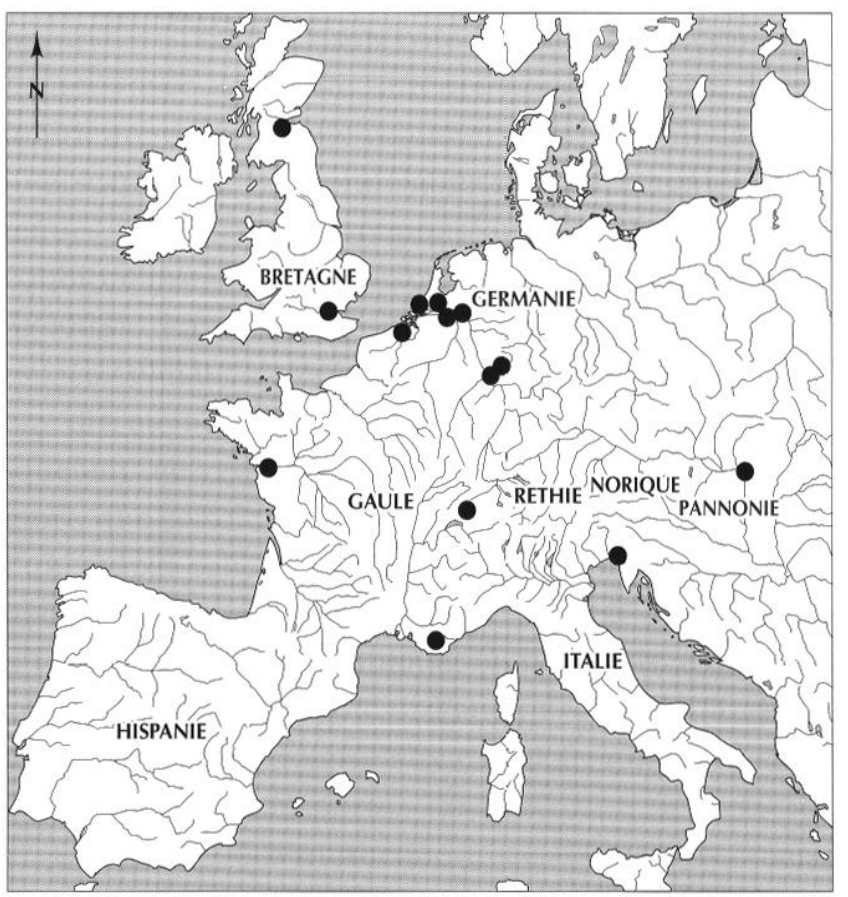

3

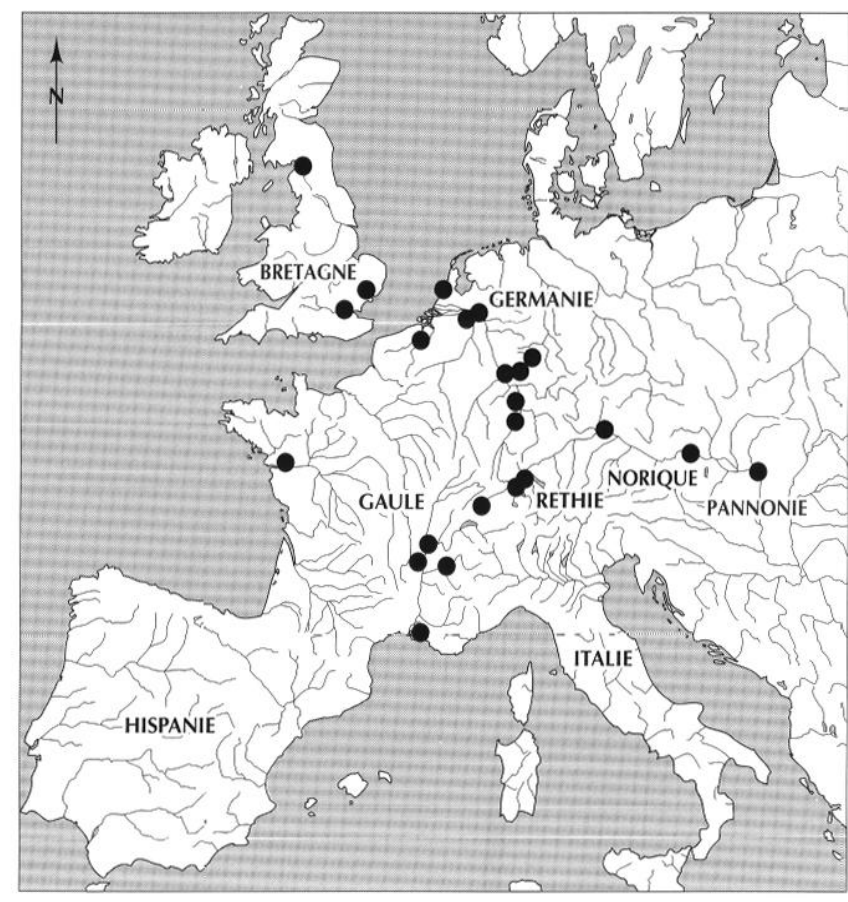

2

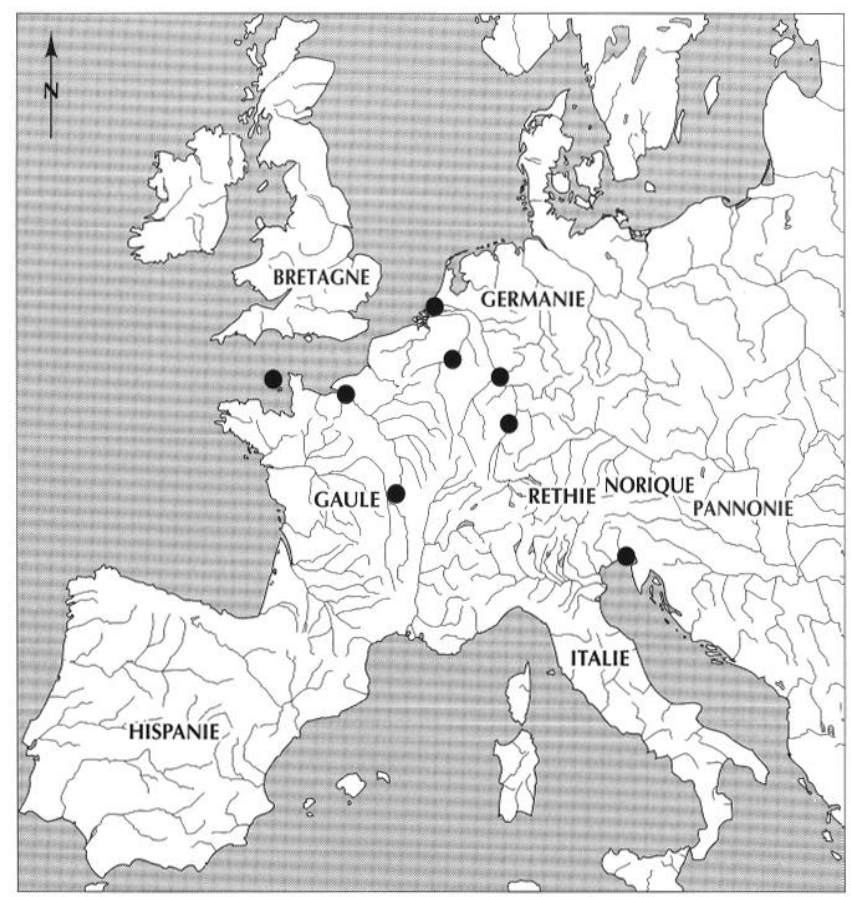

4

Fig. 102 - Cartes de répartition des tonneaux selon la chronologie :

1, Ir s. avani J.-C. ; 2, Ir s. après J.-C. ; 3, II s. aprè̀s J.-C. ; 4, III et IV s. après J.-C. (fond de carte (C M. Feugère, M. Py, CNRS, 1994). 
La carte de leur répartition au $\mathrm{I}^{\mathrm{er}} \mathrm{s}$. avant notre ère (fig. $102, n^{\circ} 1$ ) ne révèle pour le moment aucune découverte en Gaule centrale, et leur diffusion apparaît limitée aux axes de navigation fluviale et maritime. Les témoins les plus anciens, datés de La Tène finale, proviennent de Manching (T193) ${ }^{213}$ sur le Danube et de Mortantambe (T53) en Charente-Maritime ${ }^{214}$. Puis, à l'époque augustéenne, avec la création de camps sur le limes germanique, l'importation de foudres est attestée pour l'approvisionnement des troupes en vin ou en bière (Tchernia, 1997, p. 123), comme à Oberaden (T125 à T162) où l'on en a retrouvé trente-huit. À Fréjus, les fouilles de l'Argentière ont livré des douelles (T75) dans un contexte contemporain de la transformation du site en port militaire par Auguste. On possède également des douelles de la fin du siècle à Blain (T48), dans l'estuaire de la Loire.

$\mathrm{Au} \mathrm{I}{ }^{\mathrm{cr}} \mathrm{s}$. de notre ère, les indices se multiplient : on remarque clairement une distribution sur les axes du Rhône, du Rhin et du Danube, vers les camps militaires, et quelques exemplaires en Bretagne et près de l'estuaire de la Loire (fig. 102, $n^{\circ}$ 2).

Pour le siècle suivant, on constate toujours la présence de tonneaux sur lc limes rhénan, en Bretagne et dans l'embouchure de la Loire ; en revanche, la vallée du Rhône ne produit aucun témoignage pour cette période, ce qui est assez spectaculaire en comparaison avec le siècle précédent (fig. 102, $\mathrm{n}^{\circ}$ 3). Deux découvertes sont, de plus, signalées sur la côte méditerranéenne: d'une part, un jable à Pignans dans le Var (T74), et, d'autre part, la seule pièce connue pour la Cisalpine, dans une épave de la fin du $\mathrm{II}^{\mathrm{e}} \mathrm{s}$. ou du début du III $\mathrm{s}$. située à quelques milles de l'île de Grado (T202).

La répartition des découvertes de tonneaux aux $\mathrm{III}^{\mathrm{e}}$ et $I^{e}$ s. semble poursuivre la tendance amorcée au II $^{\mathrm{e}} \mathrm{s}$. (fig. 102, n ${ }^{\circ} 4$ ). En effet, les témoignages sont de plus en plus rares, ce qui paraît en contradiction avec l'emploi probablement de plus en plus commun du tonneau, mais qui peut s'expliquer par l'abandon de sa réutilisation dans les puits.

213. Les numéros renvoient au tableau $\mathrm{V}$ (p. 194-201), synthèse du catalogue détaillé des découvertes de tonncaux (Marlière, 2000 et à paraitre).

214. Les deux fonds découverts à Mortantambe sont cependant à considérer avec réserve ; $c$ en effet, aucune douelle n'ayant été conservée, rien ne permet d'affirmer s'ils appartenaient à un tonnelet ou à deux seaux.
L'archéologie ne présente en effet qu'une partie des données sur les tonneaux, puisque leur réutilisation dans les puits, qui nous fournit l'essentiel de la documentation, se concentre dans les camps militaires et semble disparaître au cours du $\mathrm{II}^{\mathrm{e}} \mathrm{s}$. On connaît donc à peine les tonneaux hors des contextes militaires et au-delà du début du III ${ }^{e}$.

\section{APPROCHE TYPOLOGIQUE DU TONNEAU}

Le nombre relativement important de vestiges permet, par ailleurs, de proposer une approche typologique. Une quarantaine d'individus parmi les tonneaux enregistrés présentent les deux paramètres que nous avons retenus: la hauteur (parfois restituée quand au moins une des douelles est conservée sur plus de la moitié de la hauteur) et le rapport d'élancement.

Le plus petit des tonneaux conservés est celui de Grenoble (T70), haut de $24,3 \mathrm{~cm}$ seulement, tandis que le plus grand provient d'un puits de Saalburg (T164) et atteint $216 \mathrm{~cm}$. Comme nous l'avons déjà évoqué, la plupart des tonneaux ont été découverts dans des puits. Il s'agit donc nécessairement de pièces de grande taille, ayant un diamètre au bouge compris entre 70 et $100 \mathrm{~cm}$. Les quelques tonneaux mis au jour dans d'autres contextes sont de dimensions nettement inférieures, comme les barriques de l'épave de Guernesey (T30 à T43) et celle réutilisée pour le captage de l'eau à Avenches (T77).

Le rapport d'élancement (H/D), c'est-à-dire la hauteur divisée par le diamètre au bouge, des tonneaux découverts en fouille se situe entre 1,03 à Vechten (T107) et 2,53 à Oberaden (T150).

La confrontation de ces paramètres a permis de distinguer cinq groupes (fig. 103).

Le premier rassemble les barriques dont la hauteur est comprise entre $24,3 \mathrm{~cm}$ et $50 \mathrm{~cm}$. Il est illustré par les cinq tonnelets de Bar Hill (T1), Grenoble (T70) et Guernesey (T30, T31 et T32); on peut ajouter à cette liste deux tonnelets, celui du Bernard (T52), pour sa petite capacité de 8 litres, et l'hypothétique tonnelet de Mortantambe (T53), dont les fonds présentent un diamètre d'environ $16-17 \mathrm{~cm}$. On peut également rapprocher de cette première série les représentations de tonnelets du cippe funéraire de l'îlot Saint-Jacques de 

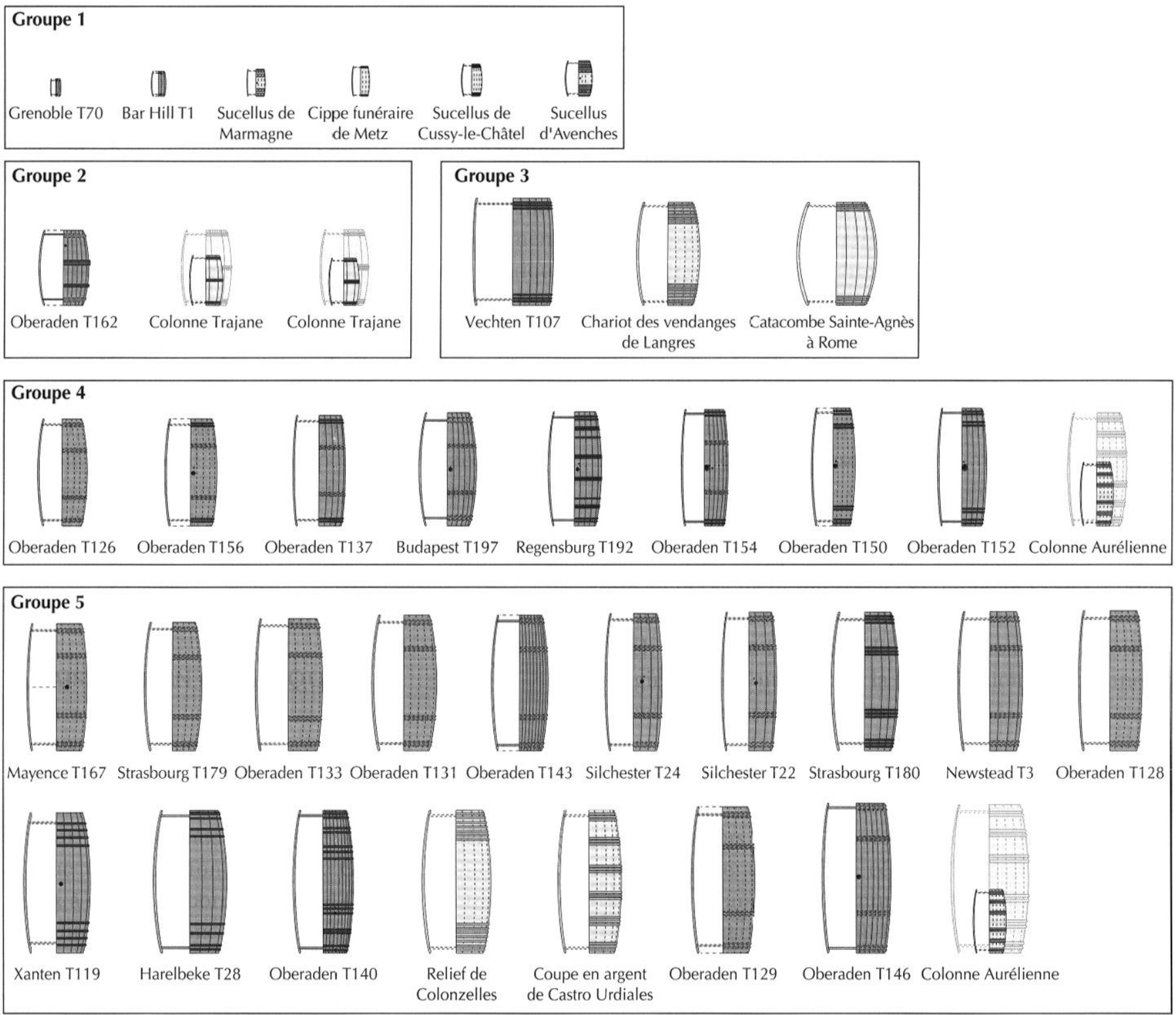

Fig. 103 - Présentation des restitutions graphiques des tonneaux classées par groupes typologiques.

Metz ${ }^{215}$, des Sucellus de Marmagne ${ }^{216}$, de Cussy-leChâtel ${ }^{217}$ et d'Avenches ${ }^{218}$. Bien qu'il se dégage manifestement du lot, cet ensemble n'est pas des plus homogènes: les rapports d'élancement s'étendent en effet de 1,22 à 1,68 et les capacités de 2,5 à 53 litres. En

215. Nerzic, 1989, p. 254 ; Faas, 1987, p. 118-119, ${ }^{\circ} 57$.

216. Thévenot, 1953, p. 293-294.

217. Espérandieu 2025.

218. Espérandieu 5392. règle générale, cette famille se caractérise par la hauteur, inférieure à $50 \mathrm{~cm}$.

Le deuxième groupe n'est constitué que d'un tonneau d'Oberaden (T162) et de deux représentations de tonneaux de la colonne Trajane ${ }^{219}$, mais il apparaît comme un véritable ensemble. Bien que les paramètres restitués des images de tonneaux de la colonne Trajane soient de dimensions inférieures, probablement à cause du manque de place dans la composition, les proportions

219. Chevallier, 1976, p. 86-87 ; Malissard, 1976, p. 57. 
sont les mêmes : ce sont des barriques peu élancées et d'une contenance probablement au-dessous de 200 litres.

Le troisième groupe est celui des foudres des chariotsciternes, que nous connaissons grâce à l'iconographie, notamment le relief du chariot des vendanges de Langres ${ }^{220}$ et une peinture de la catacombe Sainte-Agnès à Rome ${ }^{221}$. Il s'agit d'énormes tonnes hautes de 140 à $150 \mathrm{~cm}$, d'aspect tassé et d'une contenance atteignant les 1200 litres. Parmi les tonneaux que les fouilles nous ont livrés, la volumincuse futaille de Vechten (T107) pourrait très bien entrer dans cette famille.

Avec le quatrième groupe, cette typologie prend une tournure assez. intéressante. Il s'agit d'un ensemble homogène de fûts " en flûte " provenant d'Oberaden (T126, T137, T150, T152, T154, et T156), de Regensburg (T192) et de Budapest (T196 et T197), possédant une hauteur comprise entre 150 et $165 \mathrm{~cm}$, une contenance de 460 à 620 litres, et un élancement très prononcé de 1,85 à 2,53 . Nous avons donc un type de tonneau particulier qui jusqu'à maintenant n'est attesté que dans des contextes militaires.

Enfin, la cinquième famille ne diffère de la précédente que par la taille : il s'agit des grands foudres dont la hauteur varie de 178 à $216 \mathrm{~cm}$, le rapport $\mathrm{H} / \mathrm{D}$ de 1,85 à 2,35 et la capacité de 750 à 1440 litres, et dont la diffusion est limitée au limes rhénan et à la Bretagne. Le relief de Colonzelle (Drôme) ${ }^{222}$ et une coupe en argent de Castro Urdiales (Espagne) ${ }^{223}$ représentent ce type de tonnes.

Les représentations de tonneaux de la colonne Aurélienne ${ }^{224}$, dont les paramètres restitués selon la taille des personnages (estimée aux alentours de $170 \mathrm{~cm}$ ) sont de dimensions inférieures, probablement en raison du manque de place sur le relief, offrent un rapport d'élancement analogue à ceux des fûts de ces deux derniers groupes. Cette similitude constitue un point supplémentaire en faveur de l'hypothèse de leur destination militaire.

Cinq familles, caractérisées par leur hauteur, leur élancement et leur capacité, se dégagent donc; la diffusion de trois d'entre elles, les groupes 2, 4 et 5, est

220. Molin, 1984 ; Laubenheimer, 1990, p. 149.

221. Tchernia, 1986a, fig. 5.1.

222. Lspérandicu 6779 et 8021 ; Laubenhcimer, 1990, p. 152.

223. Rostovtzelf, 1971.

224. Caprino et al., 1955, p. 81-92. a priori limitée aux camps militaires de Bretagne et des limes rhénan et danubien.

\section{LE TONNEAU EN GAULE}

L'invention $\mathrm{du}$ tonneau est traditionnellement attribuée aux Gaulois sur la base de preuves assez minces qui, en fait, ne laissent en rien présumer de son origine. Son apparition n'est pas datée, mais il est déjà connu en Gaule à l'époque de César, puisque ce dernier évoque sa présence à Marseille dans la Guerre civile (II, 11, 2) et qu'Hirtius le cite à son tour dans la Guerre des Gaules (VIII, 42, 1), à l'occasion du siège d'Uxellodunum, dans le sud-ouest de la France. Tout au plus possède-t-on quelques indices d'utilisation précoce, à l'époque de La Tène, à Manching, où ont été mis au jour un tonneau carbonisé et peut-être des empreintes de tonneaux dans des fosses, ainsi qu'à Mortantambe, comme nous l'avons formulé plus haut.

Récemment, une hypothèse proposée par A. Desbat (1997) a donné au tonneau une origine étrusque. La technique d'assemblage des douclles pour les cuves était en effet connue des Étrusques comme l'attestent certaines peintures de tombes qui présentent sans ambiguité des cuves vinaires cerclées, notamment celle des Jongleurs à Tarquinia. Cette interprétation fournit des éléments de réponse recevables, mais soulève également de nombreuses questions, puisque le passage de la cuve au tonneau implique l'acquisition d'une autre technique, celle du cintrage, dont les origines et la diffusion restent à éclaircir.

À l'époque impériale, l'usage du tonneau, probablement inventé pour la bière, si l'on accepte son origine gauloise, s'étend au transport du vin, parallèlement au développement de la vigne en Gaule. Un faisceau d'indices convergents nous conduit à proposer l'hypothèse de l'existence de plusieurs grands centres de tonnellerie gaulois répartis, d'une part, dans la région lyonnaise pour le reconditionnement de vins importés en amphores ou en dolia d'Italie, d'Hispanic ou de Narbonnaise, et destinés aux troupes stationnées sur le limes, et, d'autre part, dans les régions où la viticulture s'est implantée à partir de la fin du $\mathrm{I}^{\mathrm{er}} \mathrm{s}$. avant notre ère. Notre argumentation se fonde sur les essences des bois utilisés, sur la typologie proposée précédemment, sur les noms d'artisans fournis par les marques, sur la localisa- 
tion de ces marques et des fers à marquer, et sur celle des monuments funéraires de tonneliers.

Les découvertes de haches-marteaux (sorte de hache dont le tranchant est remplacé par des lettres en relief servant à frapper la marque du tonnelier), qui semblent réservées exclusivement à l'usage des tonneliers (au contraire des autres outils qui peuvent aussi bien faire partie de ceux que manient les autres artisans du bois) ${ }^{225}$, se limitent à l'axe Rhône-Saône-Rhin (fig. 104). À l'inverse, la distribution des signacula, poinçons utilisés pour les marques au fer rouge, est bien plus large, puisqu'ils proviennent en particulier de sites de l'axe Rhône-Doubs-Danube, mais également de Mayence, de Londres et des environs d'Amiens. Les signacula mis au jour dans des régions où par ailleurs aucune hachemarteau ou aucun monument funéraire n'a été répertorié, comme à Londres, Amiens et sur les rives du Danube, n'étaient peut-être pas liés à une tonnellerie mais plutôt à un négociant en vins ou en bière, qui apposait sa marque sur les tonneaux, ou même à d'autres artisanats, ceux du cuir ou du bois par exemple.

Les monuments funéraires, qui se présentent ici sous la forme de reliefs et d'épitaphes, semblent à première vue dispersés un peu partout en Gaule et en Cisalpine, et au demcurant, pour la plupart, à des endroits où aucun outil n'a encore été découvert. Ces monuments sont en réalité situés à des points stratégiques du commerce à l'époque impériale et dans des secteurs où la production viticole a réclamé la fabrication d'emballages.

\section{LA VALLÉE DU RHÔNE}

La zone de production ${ }^{226}$ susceptible d'être la plus ancienne, mais aussi la plus importante, est celle qui se serait implantée dans la vallée du Rhône dès la fin du $\mathrm{I}^{\mathrm{er}} \mathbf{s}$. avant notre ère. La présence de tonneliers y est attestée par une épitaphe mise au jour à Rochemaure ${ }^{227}$, mais aussi par une hache-marteau provenant de SainteColombe et un signaculum trouvé à Lyon (fig. 104). Les fouilles ont également révélé la présence de tonneaux

\footnotetext{
225. Desbat, 1997, p. 115.
}

226. On entend ici par "zone de production " le regroupement de plusieurs ateliers de tonnellerie, en opposition aux ateliers isolés, liés à un usage local.

227. CIL XII, 2669. pour la plupart du I ${ }^{\text {er }}$ s. à Lyon (T63 à T67), SaintRomain-en-Gal (T68), Vienne (T69), Grenoble (T70), ct plus au sud à Glanum (T72) et à Fos-sur-Mer (T73), quoique ce dernier ait contenu du poisson et n'entre pas par conséquent dans le raisonnement qui suit.

Deux facteurs principaux paraissent en effet avoir été à l'origine de la fabrication de tonneaux dans la région lyonnaise. Le premier est la culture de l'Allobrogica, cépage dont les viticulteurs de Vienne ont tiré un grand prestige dès la fin du $\mathrm{I}^{\mathrm{er}} \mathrm{s}$. avant notre ère ${ }^{228}$ et auquel on ne peut associer que très peu d'ateliers d'amphores vinaires. Il est donc tout à fait envisageable que le vin des Allobroges ait voyagé en tonneaux plutôt qu'en amphores (André, Levadoux, 1964 ; Tchernia, 1997, p. 125-126).

Le second emploi des tonneaux lyonnais serait le reconditionnement de vins de qualité moyenne, importés en dolia, et destinés en particulier aux troupes stationnées sur le limes germanique (Martin-Kilcher, 1994 ; Desbat, 1997 ; Tchernia, 1997). A. Tchernia étaie cette hypothèse par le fait que l'époque des premières épaves à dolia: que l'on peut situer vers 20 avant notre ère, et dont fait partie l'épave du Grand-Ribaud D, datée de la dernière décennie avant notre ère ${ }^{229}$, coïncide avec le moment où Auguste envoie un grand nombre de troupes sur le limes rhénan afin de conquérir la Germanie. C'est à cette occasion que sont créés les camps de Neuss (en 16 avant notre ère), de Xanten (en 14 ou 13 avant notre ère) et d'Oberaden (vers 12 avant notre ère) par exemple, dans lesquels on a relevé la présence de nombreux tonneaux. À l'inverse on y a recueilli un nombre infime d'amphores vinaires en comparaison avec celui des amphores à huile de Bétique (Tchernia, 1997, p. 126). Le rapprochement ne s'arrête pas là, puisque c'est toujours durant cette période, entre 20 et 15 avant notre ère, que l'on commence à fabriquer à Lyon des amphores imitant la forme des amphores à poisson de Bétique et dont on suppose qu'elles étaient destinées au reconditionnement des conserves de poisson importées en vrac de cette région de l'Espagne (Dangréaux et al., 1992).

Pour S. Martin-Kilcher, les tonneaux expédiés sur le limes germanique à l'époque augustéenne, notamment à

228. Dion, 1959, p. 118-121.

229. Hesnard et al., 1988. 


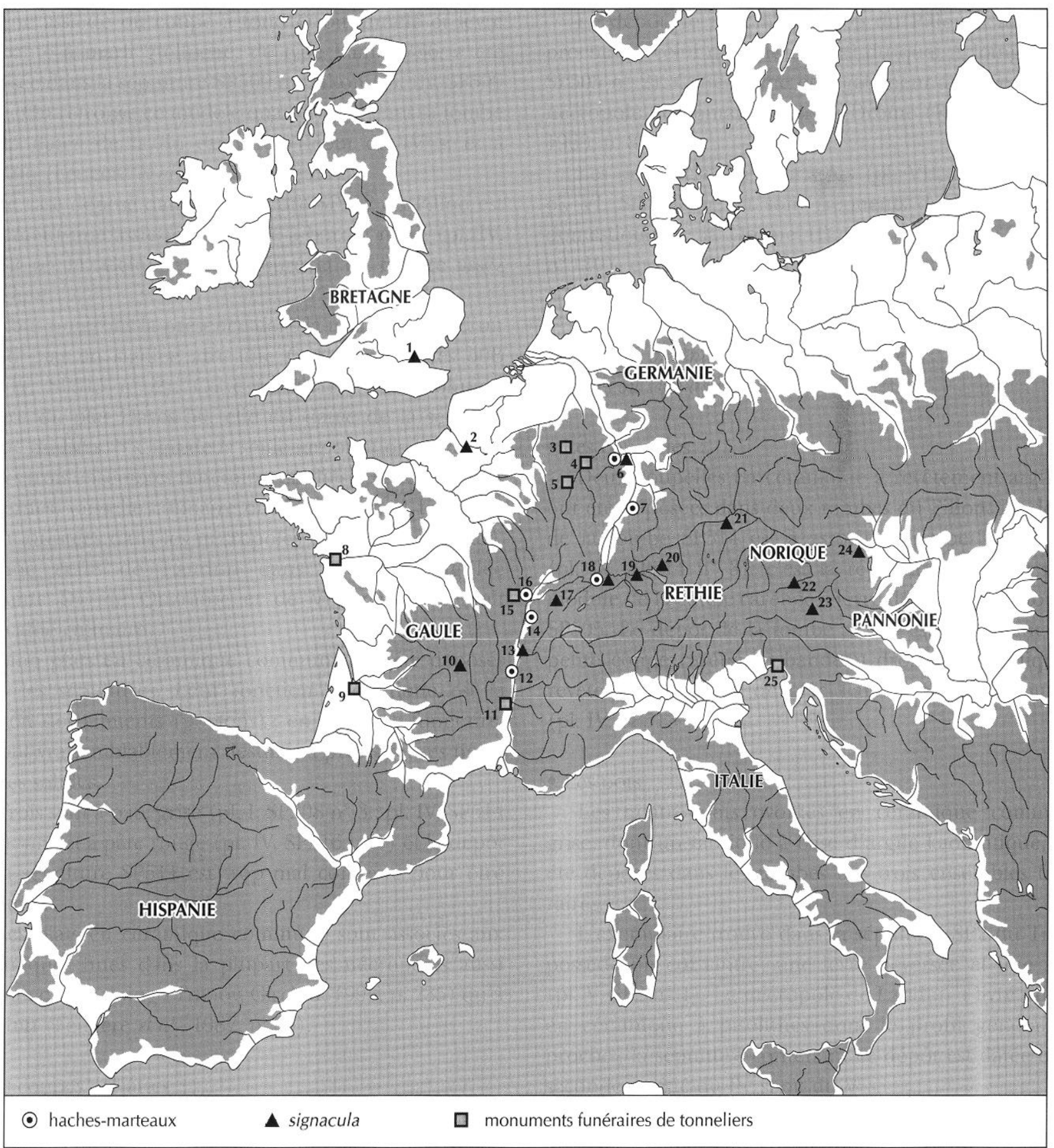

Fig. 104 - Carte de répartition des outils et des monuments funéraires de tonneliers : 1, Londres; 2, Amiens ; 3, Berbourg; 4, Trêves ; 5 , Metz ; 6, Mayence ; 7, Heidelberg ; 8, Nantes ; 9, Bordeaux ; 10, Maringues ; 11, Rochemaure ; 12, Sainte-Colombe ; 13, Lyon ; 14, Verdun-surle-I)oubs ; 15, Autun; 16, Pontailler ; 17, Pupillin ; 18, Augst ; 19, Dägerlen ; 20, Bregenz ; 21, Eining ; 22, Enns ; 23, Magdalensberg ; 24, Baden; 25, Aquilée (fond de carte () M. Feugère, M. By, CNRS, 1994).

Oberaden, peuvent également contenir du vin du sud de la Gaule, la fabrication d'amphores étant à cette époque minime en Narbonnaise, comme le montre l'approvisionnement des camps militaires (Martin-Kilcher, 1994).
Les noms fournis par les marques, l'étude des essences, la répartition et la chronologie des hachesmarteaux et des marques frappées, ainsi que la typologie confortent cette hypothèse. 
En ce qui concerne les marques, l'origine rhodanienne du foudre d'Harelbeke (T28) est avancée en 1961 par M. Renard, qui appuie son hypothèse sur les noms Gauius (marque frappée MGAV), Seuerus (marque frappée L. SEV) et Vitalis (graffite), connus dans cette région. Parallèlement, J. H. F. Bloemers trace une carte de répartition des nomina commençant par Dun, afin d'identifier le lieu d'origine d'un tonneau de Rijswijk (T105) portant la marque frappée M. DVN, et parvient à circonscrire une zone s'étendant de la région de Toulouse à la Rhétie en passant par la vallée du Rhône (Bloemers ed., 1978). Enfin, B. Galsterer attribue aux noms livrés par les marques d'Oberaden une origine gauloise ou d'Italie du Nord (Galsterer, 1992).

Par ailleurs, E. Frison, J. Viérin et C. Léva proposent pour le foudre d'Harelbeke (T28) une origine préalpine au vu des deux essences révélées par les analyses des douelles, le Sapin argenté et le Mélèze d'Europe, et des cercles, le Noisetier, ces trois essences ne croissant effectivement ensemble que dans cette région (Frison, 1961; Viérin, Léva, 1961). Or, l'identification du Mélèze est problématique, cette essence pouvant aisément être confondue avec l'Épicéa, en particulier lorsque les bois sont anciens. W. A. Casparie (1978) préfère pour cette raison proposer ccttc seconde essence pour le tonneau d'Harelbeke, et soumet l'hypothèse d'une origine rhodanienne pour l'ensemble des tonneaux présentant le Sapin et l'Épicéa ${ }^{230}$, qui est bien attesté dans la région de Lyon (Argant, 1995). Ces tonneaux n'ont par ailleurs été repérés que dans des camps militaires du nord de la vallée du Rhin.

Le fait est que la plupart de ces tonneaux présentant à la fois le Sapin argenté et l'Épicéa portent des marques frappées. Comme nous l'avons déjà évoqué précédemment, l'usage de la hache-marteau paraît se limiter aux vallées du Rhône, de la Saône et au sud de la vallée du Rhin, et les marques frappées ne concernent que des foudres trouvés dans des puits du nord de la vallée du Rhin, dans des camps militaires, dont Oberaden et Xanten, pendant une période comprise entre 19 avant notre ère et la fin du $\mathrm{I}^{\mathrm{er}}$ ou le début du $\mathrm{II}^{\mathrm{e}} \mathrm{s}$. de notre ère. Il est donc déjà possible de faire un lien entre la vallée du Rhône, qui serait le lieu de production, et les camps du limes rhénan, mais on peut pousser plus avant cette hypothèse.

230. Les tonneaux n'offrant en revanche que le Sapin, et cela concerne approximativement la moitié des exemplaires analysés, peuvent provenir de n'importe quelle région montagneuse.
Si les marques frappées n'apparaissent effectivement que sur les tonneaux mis au jour dans les camps de la partie septentrionale de la vallée du Rhin, elles semblent se limiter de plus aux tonneaux des cinquième et sixième groupes de la typologie que nous avons proposée. Seuls cinq tonneaux portant des marques frappées ont pu s'intégrer à notre classement, les publications des autres ne fournissant pas assez de paramètres pour une mise en série. L'un d'entre eux, provenant d'Oberaden (T154), correspond à la cinquième famille, celle des fûts en flûte, et les quatre autres, d'Harelbeke (T28), Xanten (T119 et T121) et Oberaden (T146), se rangent dans la famille des grands foudres, la sixième.

Tout porte donc à croire que Lyon et sa région furent un centre important de production de tonneaux, au moins pendant la période augustéenne et tout le ${ }^{\mathrm{Ir}} \mathrm{s}$. de notre ère.

\section{BORDEAUX}

Bordeaux et son terroir apparaissent chronologiquement comme le deuxième centre important de tonnellerie. Deux monuments funéraires de tonneliers sont connus à Bordeaux même. Le premier est un fragment de stèle conservé au musée d'Aquitaine montrant le tonnelier tenant une aissette et s'appuyant sur une barrique ( $c f$. fig. 83, supra, p. 159) (Espérandieu, 1907-1949, 1112 ; Laubenheimer, 1990, p. 148). Le second est une épitaphe trouvée en 1756 (CIL XIII, 1, 1, 744). En ce qui concerne la présence de tonneaux dans la région, des douelles et un fond de tonneau ont été mis au jour à Saintes, au nord de Burdigala (T54 et T55).

Ces pièces sont indubitablement à mettre en relation avec la culture de la vigne, la fameuse Biturica, dans le Bordelais dès le début du ${ }^{\mathrm{er}} \mathrm{s}$. de notre ère (Dion, 1959, p. 121-126). Il est de plus intéressant de noter que cette région n'offre que peu d'ateliers d'amphores (Bcrthault, 1992, p. 94). L'importance commerciale du port de Bordeaux, en contact à la fois avec la Méditerranée par la Garonne et avec la Bretagne par l'Atlantique, est aussi à prendre en considération pour la diffusion de ce vin.

\section{NANTES}

À Nantes, la présence de l'épitaphe d'un tonnelier (cuparius) trouvée en 1887 s'accorde très bien avec l'importance que pouvait avoir l'estuaire de la Loire au 
niveau commercial (CIL XIII, 1, 1, 3104). La présence de tonneaux est parallèlement démontrée dans la région proche par des découvertes dont les datations sont comprises entre la fin du $\mathrm{I}^{\mathrm{cr}} \mathrm{s}$. avant notre ère et la fin du II $^{c}$ s. à Blain (T48 et T49), Rezé (T50 et T51) et Le Bernard (T52). La viticulture ne serait attestée dans la région de Nantes qu'à partir du $\mathrm{VI}^{\mathrm{e}} \mathrm{s} .{ }^{231}$ par les textes, mais l'archéologie tend à montrer un développement plus précoce ${ }^{232}$. Cependant, la position seule de ce site peut justifier la présence d'une tonnellerie, et pourquoi pas dans le dessein de conditionner des productions locales comme le vin et le poisson?

\section{LA BOURGOGNE}

À Autun, la présence de tonneaux (T59 à T62) et de la stèle funćraire d'un tonnelier ${ }^{233}$, représenté avec un tonnelet et un foret, ainsi que de douelles dans un puits remblayé à la fin du IV $\mathrm{s}$. à Champallement (T58), est à mettre en relation avec le vignoble bourguignon. La culture de la vigne y est attestée par le Panégyrique de $312^{234}$, mais la production d'amphores à Gueugnon pourrait faire remonter cette datation au ${ }^{\mathrm{er}} \mathrm{s} .{ }^{235}$. Il ne nous semble pas prudent de prendre en considération les outils de tonneliers pour cette région, leur datation nous étant inconnue et l'emploi des haches-marteaux étant probablement délaissé à cettc ćpoque.

\section{LE PAYS MOSELLAN}

Enfin, au III ${ }^{\mathrm{e}}$ s., la ville de Trèves fait l'objet de faveurs impériales et finit par devenir capitale d'Empire en 293. L'expansion de la cité s'accompagne de l'apparition d'une production viticole locale, et par conséquent d'unc fabrication d'emballages, l'abondance de vin étant nécessaire au luxe nouvellement introduit dans la région.

Le pays mosellan, outre les nombreuses représentations de commerce de tonneaux, offre quatre

231. Dion, 1959, fig. 9.

232. Voir infra, la conclusion de J.-P. Brun et de F. Laubenheimer.

233. Espérandieu, 1907-1949, 1882.

234. Dion, 1959, p. 139-147 ; Laubenhcimer, 1990, p. 149-154.

235. Laubenheimer, 1986b. monuments funéraires de tonneliers: une stèle à Berbourg ${ }^{236}$, conservée au musée d'Histoire et d'Art de Luxembourg, une épitaphe à Trèves ${ }^{237}$, qui semble être celle de l'épouse d'un cuparius-saccarius, tonnelier et fabricant de sacs, et deux reliefs à Metz, le cippe funéraire de l'îlot Saint-Jacques ${ }^{238}$, dont les deux registres montrent le sciage du bois et un couple avec un tonnelet, et un relief provenant du quartier de la Citadelle ${ }^{239}$ figurant des cuves et des tonneaux.

Rares sont les tonneaux qui peuvent être directement associés au développement de la vigne sur les rives de la Moselle, mais il est vrai qu'en règle générale peu d'exemplaires du Bas-Empire nous sont parvenus. On peut toutefois évoquer les deux fûts réutilisés comme cuves à carbonate de calcium dans l'exploitation viticole d'Erden (T46 et T47, cf. supra, p. 173), dont la période d'activité s'étend du milieu du III' $s$. au début du Ves., et les deux tonneaux du $\mathrm{III}^{e}$ s., découverts à Strasbourg (T183 et T184).

À l'époque impériale, à côté de petites tonnelleries probablement dispersées un peu partout en Gaule et liées à la fabrication locale, voirc domestique, de la bic̀re et même du cidre, ateliers qui n'ont pas ou peu laissé de traces, de gros centres de fabrication de tonneaux ont donc été implantés, d'une part dans des régions viticoles, et d'autre part à de grands carrefours commerciaux comme Lyon, afin de reconditionner dans des contencurs adaptés au trafic fluvial le vin destiné aux troupes.

\section{L'ITALIE, L'HISPANIE ET LA BRETAGNE}

Bien que les témoignages n'y soient pas aussi nombreux qu'en Gaule, l'usage du tonneau est pourtant démontré dans les contrées voisines.

En Italie, la présence du tonncau cst attestée dès le $\mathrm{I}^{\mathrm{er}}$ s. de notre ère par les textes (Frontin, Stralagemata, III, 14, 3; Strabon, Géographie, V, 1, 8), par un bas-relief conscrvé à Rome (Tchernia, 1986a, p. 288 ct fig. 2) et par deux stc̀les funéraires, celle du muletier Rinnius Novicius

236. lispćrandieu 4221 ; Wilhclm, 1974, p. 42, n 289.

237. CIL XIII, 1, 2, 3700.

238. Nerzic, 1989, p. 254 ; Faas, 1987, p. $118-119$, n$^{\circ} 57$.

239. Espérandieu 4327. 
à Caraglio et celle de $\mathrm{Q}$. Veiquasius Optatus à Bene Vagienna (Molin, 1984).

La production de tonneaux n'est en revanche attestée qu'au III' $s$. dans la région d'Aquilée, d'où provient la stèle funéraire d'un tonnelier nommé L. Cantius Acutus, ornée d'un tonneau et d'une aissette (ascia) et conservée au musée archéologique d'Aquilée (Berechi, 1974). Un tonneau presque contemporain de ce monument funéraire faisait partie du chargement d'un navire de commerce échoué au large de l'île de Grado, proche d'Aquilée (T202), où il avait été réutilisé en cuve de récupération de fragments de verre destinés au recyclage.

Le cas de l'Hispanie est similaire, puisque la présence des tonneaux n'y est attestée que par la patère de Castro Urdiales (Rostovtzeff, 1971), la stèle d'un marchand de vin de Badajoz (Garcia y Bellido, 1949, $\mathrm{n}^{\circ} 324$ ) et les cupae, monuments funéraires reprenant la forme du tonneau, que l'on rencontre principalement dans les régions de Barcelone et de Lisbonne (Julia, 1965; Étienne, Mayet, 2000, p. 22-58). Il est délicat de se fier uniquement aux trouvailles de tonneaux dont la fréquence dépend directement des conditions de conservation, en grande partie liées à leur remploi dans les puits; cette pratique est visiblement peu répandue dans les contrécs méridionales.

À la différence de l'Italie et de l'Hispanie, la présence du tonneau est attestée en Bretagne par des découvertes archéologiques dès le milieu du $\mathrm{I}^{\mathrm{er}} \mathrm{s}$. de notre ère à Colchester (T12) et à Londres (T14).

Deux tonneaux de Silchester (T22 et T24) présentent des caractéristiques qui les rapprochent de la production lyonnaise que nous avons évoquée plus haut : d'une part, leur appartenance au cinquième groupe de la typologie et, d'autre part, l'emploi du Sapin argenté pour les douelles.

Les quatre tonneaux trouvés à Newstead en Écosse (T2 à T5) sont datés de la seconde moitié du $\mathrm{II}^{\mathrm{c}} \mathrm{s}$.; il est tentant de mettre leur présence en relation avec l'occupation romaine qui s'établit dans sa plus forte extension jusqu'au Mont d'Écosse sous Antonin.

$$
\text { * * }
$$

On observe donc dans les cartes de répartition un grand déséquilibre entre la documentation fournie par la Gaule, la Bretagne et les vallées du Rhin et du Danube, et celle des régions plus méridionales. Cette dissemblance résulte de deux phénomènes. Il s'agit bien entendu en premier lieu des différences climatiques qui engendrent l'humidité ou la sécheresse des sédiments archéologiques, condition inévitable de la préservation ou de la disparition des vestiges ligneux. Intervient ensuite le cadre géographique et chronologique du remploi des fûts dans les puits : la plus grande part des tonneaux enregistrés provient en effet de puits construits entre la fin du I ${ }^{\mathrm{er}} \mathrm{s}$. avant notre ère et le $\mathrm{II}^{\mathrm{e}} \mathrm{s}$. de notre ère dans les camps militaires installés le long du Rhin et du Danube. Cette majorité ne peut donc pas constituer un échantillonnage exhaustif en ce qui concerne le rôle du tonneau et l'extension de son usage dans l'Occident romain.

La question de la genèse du tonneau pâtit elle aussi de la détérioration des vestiges hors des puits. Les rares exemplaires de La Tène finale qui nous sont parvenus sont actuellement les plus anciens, mais ils sont peut-être récents dans l'histoire du tonneau. En l'absence de textes et de mobilier archéologique antérieur, il est illusoire de tenter de déterminer précisément l'époque et le lieu où le tonneau a été inventé, de saisir l'instant où un simple fabricant de seaux et de cuves est devenu tonnelier grâce au concept génial du cintrage.

L'étude du tonneau au Bas-Empire se heurte elle aussi à ce problème ; il semble effectivement qu'à cette époque on ait renoncé à la réutilisation du tonneau dans les puits en tant que "cuvelage préfabriqué ". Au moment même où la barrique prend le pas sur l'amphore pour le transport des liquides, son image, paradoxalement, devient nébuleuse. Parmi les quelques futailles des III' et IV's. livrées par les fouilles, certaines semblent cependant indiquer que le Chêne remplace progressivement les conifères pour la confection des douelles et des fonds (Guernesey T30 à T43, Rezé T50, Budapest T196).

L'autre conséquence de la prédominance de découvertes dans les puits est qu'il s'agit nécessairement de tonneaux de taille imposante ; la recherche est ainsi bien moins documentée sur les futailles dont le diamètre au bouge est inférieur à $70 \mathrm{~cm}$. Cela entraîne inévitablement des répercussions dans la typologie que nous proposons: les groupes des petits tonneaux sont soit hétérogènes, soit représentés par un seul individu. Un nombre plus important de futailles de petites et moyennes dimensions nous aiderait à affiner leur classification. En revanche, la typologie des grands tonneaux s'appuie sur de nombreuses données et nous permet 
d'avancer quelques idées, notamment en ce qui concerne la destination militaire des groupes 4 et 5 .

La confrontation des marques, des essences, des vestiges liés aux tonneliers et de la typologie nous a par ailleurs permis de conforter l'hypothèse proposée par S. Martin-Kilcher (1994), A. Tchernia (1997) et A. Desbat (1997), selon laquelle les tonneaux mis au jour dans les camps militaires de Bretagne, du Rhin et du Danube avaient été assemblés dans la vallée du Rhône afin de transporter vers les troupes des denrées importées en vrac, vins, bière et probablement des salaisons de poisson, par voie fluvialc. Cette hypothèse nous convient tout au moins pour les fûts des groupes 2, 4 et 5 de notre typologie datés de l'époque augustéenne jusqu'à la fin du $\mathrm{I}^{\mathrm{er}} \mathrm{s}$. D'autres zones de production de tonneaux se manifestent dans les régions viticoles du territoire des Allobroges, du Bordelais, de la Bourgogne et du pays mosellan.

On ne peut que constater et comprendre le succès du tonneau dès la fin du $I^{\text {er }} s$. avant notre ère et la rude concurrence qu'il dut livrer à l'amphore pour le transport terrestre et fluvial. L'archéologie n'a livré qu'une épave dont le chargement était constitué de tonneaux, celle de Guernesey, datée du III ${ }^{\mathrm{e}} \mathrm{s}$. Ce type de vestige sous-marin est en effet très difficilement repérable à cause de la destruction de toutes les structures en bois émergentes. À partir des maigres bases documentaires dont nous disposons, il est très difficile d'apprécier la part prise par le tonneau dans le commerce maritime.

Les qualités techniques de la barrique (son rapport poids/contenance très intéressant, sa résistance aux chocs et aux pressions, sa maniabilité) ont fait d'elle un conteneur efficace et compétitif. Malgré tous ces avantages, elle n'a pas immédiatement remplacé l'amphore, et cela pour plusieurs raisons : parfaite pour la fermentation, elle ne serait pas adaptée au vieillissement et à la conservation des vins. Au contraire de l'amphore, le tonneau est perméable à l'air et une seconde fermentation s'y opérerait après quelque temps. Enfin, il ne faut surtout pas négliger la force de la tradition méditerranéenne, dont les amphores sont en quelque sorte le symbole. 
Tabl. V - Tableau récapitulatif des tonneaux antiques.

(p. 196-203) 
Tabl. V - Tableau récapitulatif des tonneaux antiques :

$N D$, nombre de douelles; $H$, hauteur $; D$, diamètre au bouge $; d$, diamètre aux extrémités ; $H / D$, rapporl d'élancemenl.

\begin{tabular}{|c|c|c|c|c|c|c|c|}
\hline Région & Site & $\mathbf{N}^{\circ}$ & Contexte & Datation & Description & Essence(s) douelles & Essence cercles \\
\hline \multirow[t]{24}{*}{ Bretagne } & Bar Hill & $\mathrm{T} 1$ & & & tonnelet & Chêne & \\
\hline & \multirow[t]{4}{*}{ Newstead } & $\mathrm{T} 2$ & puits 94 & $2^{\mathrm{e}}$ moitié $\|^{\mathrm{e}} \mathrm{s}$. & tonneau incomplet & Pin sylvestre & Noisetier \\
\hline & & $\mathrm{T} 3$ & puits 94 & $2^{\mathrm{e}}$ moitié $\|^{\mathrm{e}} \mathrm{s}$. & tonneau & Pin sylvestre & Boulcau \\
\hline & & $\mathrm{T} 4$ & puits 96 & $2^{\mathrm{e}}$ moitié $\|^{\mathrm{e}} \mathrm{s}$. & tonneau incomplet & Pin sylvestre & Noisetier \\
\hline & & T5 & puits 96 & $2^{\mathrm{e}}$ moitié $\|^{\mathrm{e}} \mathrm{s}$. & tonneau & Pin sylvestre & Noisetier \\
\hline & Vindolanda & T6 & & & douelles & Sapin & \\
\hline & Carlisle & $\mathrm{T} 7$ & & 70-80 de notre ère & planche de fond & & \\
\hline & Kirkby Thore & $\mathrm{T} 8$ & & & & & \\
\hline & Segontium & T9 & & & & & \\
\hline & Haverfield & $\mathrm{T} 10$ & & & & & \\
\hline & Stone & $\mathrm{T} 11$ & & & & & \\
\hline & Colchester & $\mathrm{T} 12$ & puits & 50-60 de notre ère & tonneau incomplet & & Noisetier \\
\hline & Wickford & $\mathrm{T} 13$ & & & & & \\
\hline & \multirow[t]{6}{*}{ Londres } & $\mathrm{T} 14$ & puits & 50-150 de notre ère & tonneau incomplet & & \\
\hline & & $\mathrm{T} 15$ & puits & vers 100 de notre ère & tonneau & & \\
\hline & & $\mathrm{T} 16$ & & vers 140 de notre ère & bouchon & & \\
\hline & & $\mathrm{T} 17$ & & vers 140 de notre ère & bouchon & & \\
\hline & & $\mathrm{T} 18$ & & & bouchon & & \\
\hline & & $\mathrm{T} 19$ & & & bouchon & & \\
\hline & \multirow[t]{5}{*}{ Silchester } & $\mathrm{T} 20$ & puits insula 14 & & tonneau & & \\
\hline & & $\mathrm{T} 21$ & puits insula 14 & & tonneau & & \\
\hline & & $\mathrm{T} 22$ & puits insula 17 & & tonneau & Sapin argenté & Noisetier \\
\hline & & $\mathrm{T} 23$ & puits insula 18 & & tonneau incomplet & Sapin argenté & Noisetier \\
\hline & & $\mathrm{T} 24$ & puits insula 18 & & tonneau & Sapin argenté & Noisetier \\
\hline \multirow[t]{27}{*}{ Gaule } & \multirow[t]{2}{*}{ Kuurne } & $\mathrm{T} 25$ & & & tonneau incomplet & & \\
\hline & & $\mathrm{T} 26$ & & & tonneau incomplet & & \\
\hline & Aardenburg & $\mathrm{T} 27$ & puits & $I^{\mathrm{e}} \mathrm{s}$. & tonneau incomplet & Sapin argenté/Mélèze & \\
\hline & Harelbeke & T28 & puits & $2^{\mathrm{e}}$ moitié er $\mathrm{er}$ s. & tonneau incomplet & Sapin argenté/Mélèze & Noisetier \\
\hline & Bavay & T29 & & & douelles incomplètes & & \\
\hline & \multirow[t]{14}{*}{ Guernesey } & $\mathrm{T} 30$ & épave & $\mathrm{III}^{\mathrm{e}} \mathrm{s}$. & douelle & Chêne & Noisetier \\
\hline & & $\mathrm{T} 31$ & épave & $\mathrm{III}^{\mathrm{e}} \mathrm{s}$. & douelle incomplète & Chêne & Noisetier \\
\hline & & $\mathrm{T} 32$ & épave & $\mathrm{III}^{\mathrm{e}} \mathrm{s}$ & douelle incomplète & Chêne & Noisetier \\
\hline & & $\mathrm{T} 33$ & épave & $\mathrm{III}^{\mathrm{e}} \mathrm{s}$. & douelle incomplète & Chêne & Noisetier \\
\hline & & T34 & épave & $\mathrm{III}^{\mathrm{e}} \mathrm{s}$. & douelle incomplète & Chêne & Noisetier \\
\hline & & T35 & épave & $\mathrm{III}^{\mathrm{e}} \mathrm{s}$ & douelle incomplète & Chêne & Noisetier \\
\hline & & $\mathrm{T} 36$ & épave & $\mathrm{III}^{\mathrm{e}} \mathrm{s}$. & douelle incomplète & Chêne & Noisetier \\
\hline & & $\mathrm{T} 37$ & épave & $\mathrm{III}^{\mathrm{e}} \mathrm{s}$. & douelle incomplète & Chêne & Noisetier \\
\hline & & T38 & épave & $\mathrm{III}^{\mathrm{e}} \mathrm{s}$. & douelle incomplète & Chêne & Noisetier \\
\hline & & T39 & épave & $11 \mathrm{l}^{\mathrm{e}} \mathrm{s}$ & douelle incomplète & Chêne & Noisetier \\
\hline & & $\mathrm{T} 40$ & épave & $\mathrm{III}^{\mathrm{e}} \mathrm{s}$ & douelle incomplète & Chêne & Noisetier \\
\hline & & $\mathrm{T} 41$ & épave & $11 \mathrm{I}^{\mathrm{e}} \mathrm{s}$. & douelle incomplète avec bonde & Chêne & Noisetier \\
\hline & & $\mathrm{T} 42$ & épave & $\mathrm{III}^{\mathrm{e}} \mathrm{s}$. & douelle incomplète avec poix & Chêne & Noisetier \\
\hline & & $\mathrm{T} 43$ & épave & $\mathrm{III}^{\mathrm{e}} \mathrm{s}$. & demi-couvercle de tonnelet & & \\
\hline & Rouen & $\mathrm{T} 44$ & habitat & $\mathrm{III}^{\mathrm{e}} \mathrm{s}$ & fond & & \\
\hline & \begin{tabular}{|l|} 
Reims \\
\end{tabular} & $\mathrm{T} 45$ & habitat & $\| I^{\mathrm{e}}-11 \mathrm{I}^{\mathrm{e}} \mathrm{s}$ & cercle et cannelle & & métallique \\
\hline & \multirow[t]{2}{*}{ Erden } & $\mathrm{T} 46$ & expl. viticole & milieu III e -début Ves. & négatif de tonneau & & \\
\hline & & $\mathrm{T} 47$ & expl. viticole & milieu IIIe -début Ve s. & négatif de tonneau & & \\
\hline & \multirow[t]{2}{*}{ Blain } & $\mathrm{T} 48$ & puits & fin $\mathrm{l}^{\mathrm{er}} \mathrm{s}$. avant J.-C. & douelles & & \\
\hline & & T49 & puits & $2^{\mathrm{e}}$ moitié $\mathrm{l}^{\mathrm{er}} \mathrm{s}$. & douelles & & \\
\hline & \multirow[t]{2}{*}{ Rezé } & T50 & puits & avant fin $I^{e} s$. & douelles & Chêne & \\
\hline & & T51 & puits & avant fin $\|^{e} s$. & bouchons & & \\
\hline
\end{tabular}




\begin{tabular}{|c|c|c|c|c|c|c|c|c|}
\hline ND & H & D & d & Capacité & $H / D$ & Marques & Restitution & Bibliographie \\
\hline 14 & $35 \mathrm{~cm}$ & $22 \mathrm{~cm}$ & & $8 / 8,5$ I & 1,59 & IANVARIVS & Bar Hill 1 & Collingwood, Wright, 1992 \\
\hline 17 & & & $80 \mathrm{~cm}$ & & & & & Breuer, 1920 \\
\hline 17 & $195 \mathrm{~cm}$ & $97 \mathrm{~cm}$ & $80 \mathrm{~cm}$ & $1100 I$ & 2,01 & & Newstead 3 & \\
\hline \multicolumn{9}{|l|}{17} \\
\hline \multirow[t]{7}{*}{17} & $195 \mathrm{~cm}$ & & & & & & & \\
\hline & & & & & & & & Birley, 1993 \\
\hline & & & & & & NOVIXI & & Hassall, Tomlin, 1990 \\
\hline & & & & & & & & Desbat, 1997 \\
\hline & & & & & & & & Desbat, 1997 \\
\hline & & & & & & & & Desbat, 1997 \\
\hline & & & & & & & & Desbat, 1997 \\
\hline \multirow[t]{3}{*}{17} & & $40 \mathrm{~cm}$ & & & & & & Ulbert, 1959 \\
\hline & & & & & & & & Desbat, 1997 \\
\hline & & & & & & T. C. PACA, C. P(...) & & Ulbert, 1959 \\
\hline \multirow[t]{6}{*}{17} & $180 \mathrm{~cm}$ & & & & & T. C. PACA & & \\
\hline & & & & & & & & Jones, Rhodes, 1980 \\
\hline & & & & & & & & \\
\hline & & & & & & & & \\
\hline & & & & & & & & \\
\hline & & & & & & & & Ulbert, 1959 \\
\hline \multirow[t]{2}{*}{18} & $194 \mathrm{~cm}$ & $82,5 \mathrm{~cm}$ & $67,5 \mathrm{~cm}$ & 7501 & 2,35 & AR & Silchester 22 & Fox, St John Hope, 1900 \\
\hline & & & & & & SVALINOS & & Fox, St John Hope, 1901 \\
\hline \multirow[t]{2}{*}{19} & $192 \mathrm{~cm}$ & $85 \mathrm{~cm}$ & $70 \mathrm{~cm}$ & 8001 & 2,25 & & Silchester 24 & \\
\hline & & & & & & & & Viérin, Léva, 1961 \\
\hline & & & & & & & & \\
\hline $\mathrm{au}-16$ & & & & & & & & Lebel, 1964 \\
\hline \multirow[t]{5}{*}{18} & $200 \mathrm{~cm}$ & $106 \mathrm{~cm}$ & $83,6 \mathrm{~cm}$ & 13301 & 1,88 & CTC, MGAV, TER. STEV, L. SEV, TCL. GP, VITALIS & Harelbeke 28 & Viérin, Léva, 1961 \\
\hline & & & & & & & & Viérin, Léva, 1961 \\
\hline & $50 \mathrm{~cm}$ & & & & & & & Rule, Monaghan, 1993 \\
\hline & $45 \mathrm{~cm}$ & & & & & & & \\
\hline & $48 \mathrm{~cm}$ & & & & & & & \\
\hline & & & & & & & & \\
\hline & & & & & & & & \\
\hline & & & & & & & & \\
\hline & & & & & & & & \\
\hline & & & & & & & & \\
\hline & & & & & & & & \\
\hline & & & & & & & & \\
\hline & & & & & & & & \\
\hline & & & & & & & & \\
\hline & & & & & & & & \\
\hline & & & & & & & & \\
\hline & & & & & & & & Audin, 1985 \\
\hline & & & & & & & & Frézouls, 1983 \\
\hline env. 22 & & & & & & & & Gilles, 1994 ; Gilles dir., 1995 \\
\hline & & & & & & & & \\
\hline & & & & & & & & Audin, 1985 \\
\hline & & & & & & & & \\
\hline & & & & & & & & Audin, 1985 \\
\hline & & & & & & $R, E, A$ & & \\
\hline
\end{tabular}


Tabl. V - Tableau récapitulatif des tonneaux antiques (suite).

\begin{tabular}{|c|c|c|c|c|c|c|c|}
\hline Région & Site & $\mathbf{N}^{0}$ & Contexte & Datation & Description & Essence(s) douelles & Essence cercles \\
\hline \multirow{33}{*}{$\begin{array}{l}\text { Gaule } \\
\text { (suite) }\end{array}$} & Le Bernard & T52 & puits funéraire & & tonnelet & & métallique \\
\hline & Mortantambe & T53 & habitat & La Tène finale & tonnelet (ou deux seaux ?) & Chêne & métallique \\
\hline & \multirow[t]{2}{*}{ Saintes } & T54 & & & douelles & & \\
\hline & & T55 & & & fond & & \\
\hline & Lectoure & T56 & puits 8 & après 50 avant J.-C. & fragments de bouchons & & \\
\hline & La Chapelle-Vaupelteigne & T57 & puits & & douelles & & \\
\hline & Champallement & T58 & puits & avant fin IV $\mathrm{s}$. & douelles & & \\
\hline & \multirow[t]{4}{*}{ Autun } & T59 & expl. viticole? & & négatif de tonneau avec fond & & \\
\hline & & T60 & expl. viticole? & & négatif de tonneau & & \\
\hline & & T61 & expl. viticole? & & négatif de tonneau & & \\
\hline & & T62 & expl. viticole? & & négatif de tonneau & & \\
\hline & \multirow[t]{5}{*}{ Lyon } & T63 & puits 310 & 70 de notre ère & tonneau & Sapin/Pin & Noisetier? \\
\hline & & T64 & puits 212 & 70 de notre ère & tonneau & Sapin/Pin & Noisetier? \\
\hline & & T65 & habitat & & négatif de tonneau & & \\
\hline & & T66 & habitat & & négatif de tonneau & & \\
\hline & & T67 & Saône & $\rho^{e r} s$. & bouchon & & \\
\hline & Saint-Romain-en-Gal & T68 & habitat & $\rho^{\text {er }} s$. & négatif de tonneau & & \\
\hline & Vienne & T69 & & & tonneau & & \\
\hline & Grenoble & $\mathrm{T} 70$ & puits & $\rho^{\mathrm{er}} \mathrm{s}$. & douelle et fonds de tonnelet & & \\
\hline & Lattes & $\mathrm{T} 71$ & puits 2 & Ier s. avant J.-C. & tonneau incomplet & & \\
\hline & Glanum & $\mathrm{T} 72$ & & & & & \\
\hline & Fos-sur-Mer & $\mathrm{T} 73$ & horrea & fers. & tonneau incomplet & Sapin argenté & \\
\hline & Pignans & $\mathrm{T} 74$ & & $\|^{\mathrm{e}} s$. & jable & & \\
\hline & Fréjus & $\mathrm{T} 7 \overline{5}$ & habitat & 20-10 avant J.-C. & douelles & Sapin argenté & \\
\hline & Nyon & T76 & & & & & \\
\hline & Avenches & $\mathrm{T} 77$ & captage & $70-150$ & tonneau & & \\
\hline & Kaiseraugst & T78 & & & tonneau & & \\
\hline & Windisch & T79 & & jers. & trois douelles & Sapin & \\
\hline & \multirow[t]{5}{*}{ Oberwinterthur } & $\mathrm{T} 80$ & habitat & milieu fer $s$. & tonneau incomplet avec fond & Sapin argenté & Noisetier \\
\hline & & $\mathrm{T} 81$ & habitat & milieu ler $\mathrm{s}$. & tonneau incomplet avec fond & Sapin argenté & Noisetier \\
\hline & & T82 & habitat & milieu $\mathrm{fer}^{\mathrm{e}} \mathrm{s}$. & tonneau incomplet avec fond & Sapin argenté & Noisetier \\
\hline & & T83 & habitat & milieu ler $^{\mathrm{s}}$. & douelles et planches de fond & & \\
\hline & & T84 & habitat & milieu $\mathrm{I}^{\mathrm{er}} \mathrm{s}$. & douelles et planches de fond & & \\
\hline \multirow{18}{*}{$\begin{array}{l}\text { Limes } \\
\text { rhénan }\end{array}$} & Velsen & T85 & & $1^{\text {re }}$ moitié ler s. & tonneau & Sapin argenté/Épicéa & \\
\hline & \multirow[t]{3}{*}{ Katwÿk-sur-Mer } & T86 & puits & & tonneau & & \\
\hline & & T87 & puits & & tonneau & Aulne & Noisetier \\
\hline & & T88 & puits & & tonneau incomplet & Chêne & Saule \\
\hline & \multirow[t]{4}{*}{ Valkenbourg } & T89 & puits & avant 70 de notre ère & tonneau & & \\
\hline & & T90 & puits & avant 70 de notre ère & tonneau & & \\
\hline & & T91 & puits & avant 70 de notre ère & tonneau & & \\
\hline & & T92 & puits & avant 70 de notre ère & tonneau & & \\
\hline & \multirow[t]{10}{*}{ Arentsburg - Voorburg } & T93 & puits & Ier s. avant J.-C. & tonneau incomplet & & \\
\hline & & T94 & puits & jer s. avant J.-C. & tonneau incomplet & & \\
\hline & & T95 & puits & avant III $\mathrm{s}$. & tonneau incomplet & & \\
\hline & & T96 & & & tonneau incomplet & & \\
\hline & & Т97 & & & tonneau incomplet & & \\
\hline & & T98 & & & tonneau incomplet & & \\
\hline & & T99 & & & tonneau incomplet & & \\
\hline & & T100 & & & douelle & & \\
\hline & & T101 & & & douelle & & \\
\hline & & T102 & & & douelle & & \\
\hline
\end{tabular}




\begin{tabular}{|c|c|c|c|c|c|c|c|c|}
\hline ND & $\mathrm{H}$ & D & d & Capacité & $H / D$ & Marques & Restitution & Bibliographie \\
\hline & & & & 81 & 1,52 & & & Baudry, Ballereau, 1873 \\
\hline & & & $17 \mathrm{~cm}$ & & & & & Laporte dir., 1998 \\
\hline & & & & & & MAIB & & Sciallano, 1993 \\
\hline & & & & & & & & \\
\hline & & & & & & & & Audin, 1985 \\
\hline & & & & & & & & Audin, 1985 \\
\hline & & & & & & & & Audin, 1985 \\
\hline & & & & & & & & Landriot, 1844 \\
\hline & & & & & & & & \\
\hline & & & & & & & & \\
\hline & & & & & & & & \\
\hline 18 & & $90 \mathrm{~cm}$ & & & & & & Bellon, 1995 \\
\hline \multirow[t]{6}{*}{15} & & $75 \mathrm{~cm}$ & & & & & & \\
\hline & & & & & & & & Desbat, 1991 \\
\hline & & & & & & & & \\
\hline & & & & & & VRITTI PH & & \\
\hline & & & & & & & & Desbat, 1991 \\
\hline & & & & & & & & Desbat, 1991 \\
\hline \multirow[t]{3}{*}{12} & $24,3 \mathrm{~cm}$ & $15,3 \mathrm{~cm}$ & $11 \mathrm{~cm}$ & $2,5 / 31$ & 1,58 & & Grenoble 70 & Dangréaux, 1989 \\
\hline & & $90 \mathrm{~cm}$ & $78 \mathrm{~cm}$ & & & & & Py, 1988 \\
\hline & & & & & & & & Desbat, 1997 \\
\hline \multirow[t]{7}{*}{12} & & & & & & SENNON, B(...) & & Sciallano, 1993 \\
\hline & & & & & & & & Brun, 1999 \\
\hline & & & & & & & & Brentchaloff, Rogers, 1979 \\
\hline & & & & & & & & Desbat, 1997 \\
\hline & $80 \mathrm{~cm}$ & & $45 \mathrm{~cm}$ & & & & Avenches 77 & Blanc et al., 1995 \\
\hline & & & & & & & & Desbat, 1991 \\
\hline & & & & & & & & Ulbert, 1959 \\
\hline 19 & & & $82 \mathrm{~cm}$ & & & BACCVS I, Q. ATTI GRATI, Q. AC, C & & Fellmann, 1991 \\
\hline 18 & & & $83 \mathrm{~cm}$ & & & BACCVS I, T. IVL. MVRRANI & & \\
\hline \multirow[t]{3}{*}{18} & & & $83 \mathrm{~cm}$ & & & MARNVS I, L. C. LYDI, Q. IVL, PACATI & & \\
\hline & & & & & & T. TERTI, L. ANINVS(...)MAC OU L. AVNVS(...)MAC & & \\
\hline & & & & & & Q. FL. SILVAN & & \\
\hline \multirow[t]{2}{*}{11} & & & & & & & & Desbat, 1997 \\
\hline & & & & & & & & Ulbert, 1959 \\
\hline \multirow[t]{2}{*}{19} & & & & & & & & Breuer, 1920 \\
\hline & & $72 \mathrm{~cm}$ & $62 \mathrm{~cm}$ & & & & & \\
\hline 18 & $200 \mathrm{~cm}$ & & & & & & & Ulbert, 1959 \\
\hline & & & & & & & & \\
\hline & & & & & & & & \\
\hline 18 & & & $77 \mathrm{~cm}$ & & & SI. CAV, CGS, I?SM, VICT, C?TVICT, VA. RO. V. & & Breuer, 1918 \\
\hline \multicolumn{9}{|l|}{18} \\
\hline 17 & $130 \mathrm{~cm}$ & & $75,5 \mathrm{~cm}$ & & & MMMM, SIL. R, R & & \\
\hline 17 & $140 \mathrm{~cm}$ & & $66,5 \mathrm{~cm}$ & & & MVC, CSPS?, (CSP)C & & \\
\hline 24 & & & $78 \mathrm{~cm}$ & & & CSe?DICIVs & & \\
\hline 12 & $140 \mathrm{~cm}$ & & $54 \mathrm{~cm}$ & & & C, VTE. L, (...). G. & & \\
\hline \multirow[t]{4}{*}{12} & & & $53 \mathrm{~cm}$ & & & & & \\
\hline & & & & & & G. L & & \\
\hline & & & & & & MC & & \\
\hline & & & & & & TC ou TG, C.S. AV+ & & \\
\hline
\end{tabular}


Tabl. V - Tableau récapitulatif des tonneaux antiques (suile).

\begin{tabular}{|c|c|c|c|c|c|c|c|}
\hline Région & Site & $\mathbf{N}^{\circ}$ & Contexte & Datation & Description & Essence(s) douelles & Essence cercles \\
\hline \multirow{51}{*}{$\begin{array}{l}\text { Limes } \\
\text { rhénan } \\
\text { (suite) }\end{array}$} & & $\mathrm{T} 103$ & & & douelle & & \\
\hline & & $\mathrm{T} 104$ & & & douelle & & \\
\hline & \multirow[t]{2}{*}{ Rijswijk } & $\mathrm{T} 105$ & puits & vers 200 & tonneau & Sapin argenlé/Épicéd & Noiselier \\
\hline & & T106 & puits & & tonneau & Sapin argenté/Épicéa & \\
\hline & \multirow[t]{5}{*}{ Vechten } & $\mathrm{T} 107$ & puits & & tonneau & & \\
\hline & & T108 & puits & & tonneau incomplet & & \\
\hline & & T109 & puits & & tonneau & & \\
\hline & & $\mathrm{T} 110$ & puits & & tonneau & & \\
\hline & & $\mathrm{T} 111$ & puits & & tonneau & & \\
\hline & Druten & $\mathrm{T} 112$ & & & tonneau & & \\
\hline & \multirow[t]{2}{*}{ Woerd de Ressen } & $\mathrm{T} 113$ & puits & fin $\mathrm{I}^{\mathrm{er}}$-début $\|^{\mathrm{e}} \mathrm{s}$. & & & \\
\hline & & $\mathrm{T} 114$ & puits & & & & \\
\hline & \multirow[t]{3}{*}{ Nimègue } & T115 & puits & & tonneau incomplet & Sapin rouge & \\
\hline & & T116 & puits & & tonneau incomplet & Sapin rouge & \\
\hline & & $\mathrm{T} 117$ & Wàal & & douelles & & \\
\hline & \multirow[t]{6}{*}{ Xanten } & T118 & puits I & avant milieu $\mathrm{I}^{\mathrm{er}} \mathrm{s}$. & tonneau incomplet & Sapin argenté/Épicéa & Noisetier \\
\hline & & T119 & puits I & avant milieu $\mathrm{P}^{\mathrm{er}} \mathrm{s}$. & tonneau & Sapin argenté/Épicéa & Noisetier \\
\hline & & $\mathrm{T} 120$ & puits I & avant milieu $\mathrm{I}^{\mathrm{er}} \mathrm{s}$. & tonneau incomplet & Sapin & \\
\hline & & $\mathrm{T} 121$ & puits III & avant milieu $\mathrm{I}^{\mathrm{er}} \mathrm{s}$. & tonneau incomplet & Sapin argenté/Épicéa & Noisetier \\
\hline & & $\mathrm{T} 122$ & puits III & avant milieu $\mathrm{I}^{\mathrm{er}} \mathrm{s}$. & tonneau incomplet & Pin & Noisetier \\
\hline & & $\mathrm{T} 123$ & puits insula 39 & avant milieu II $\mathrm{s}$. & tonneau incomplet & & \\
\hline & Mülheim & $\mathrm{T} 124$ & & & & & \\
\hline & \multirow[t]{29}{*}{ Oberaden } & $\mathrm{T} 125$ & puits 4 & vers 11 avant J.-C. & tonneau incomplet & Sapin argenté & \\
\hline & & $\mathrm{T} 126$ & puits 4 & vers 11 avant J.-C. & tonneau & Sapin argenté & \\
\hline & & $\mathrm{T} 127$ & puits 5 & vers 11 avant J.-C. & tonneau incomplet & Sapin argenté & \\
\hline & & $\mathrm{T} 128$ & puits 5 & vers 11 avant J.-C. & tonneau & Sapin argenté & \\
\hline & & $\mathrm{T} 129$ & puits 7 & vers 11 avant J.-C. & tonneau & Sapin argenté & \\
\hline & & $\mathrm{T} 130$ & puits 9 & vers 11 avant J.-C. & tonneau incomplet & Sapin argenté & \\
\hline & & $\mathrm{T} 131$ & puits 9 & vers 11 avant J.-C. & tonneau & Sapin argenté & \\
\hline & & $\mathrm{T} 132$ & puits 17 & vers 11 avant J.-C. & tonneau incomplet & Sapin argenté & \\
\hline & & $\mathrm{T} 133$ & puits 17 & vers 11 avant J.-C. & tonneau & Sapin argenté & \\
\hline & & $\mathrm{T} 134$ & puits 26 & vers 11 avant J.-C. & tonneau incomplet & Sapin argenté & \\
\hline & & $\mathrm{T} 135$ & puits 29 & vers 11 avant J.-C. & tonneau incomplet & Sapin argenté & \\
\hline & & T136 & puits 29 & vers 11 avant J.-C. & tonneau & Sapin argenté & \\
\hline & & T137 & puits 1963-I & vers 11 avant J.-C. & tonneau incomplet & Sapin argenté & Noisetier \\
\hline & & $\mathrm{T} 138$ & puits 1963-II & vers 11 avant J.-C. & tonneau incomplet & Sapin argenté & Noisetier \\
\hline & & T139 & puits 1963-II & vers 11 avant J.-C. & tonneau incomplet & Sapin argenté & Noisetier \\
\hline & & $\mathrm{T} 140$ & puits $77 / 102$ & vers 11 avant J.-C. & tonneau & Sapin argenté & Noisetier \\
\hline & & $\mathrm{T} 141$ & puits $78 / 34$ & vers 11 avant J.-C. & tonneau incomplet avec fonds & Sapin argenté & \\
\hline & & $\mathrm{T} 142$ & puits $78 / 44$ & vers 11 avant J.-C. & tonneau incomplet & Sapin argenté & \\
\hline & & $\mathrm{T} 143$ & puits $78 / 44$ & vers 11 avant J.-C. & tonneau & Sapin argenté & \\
\hline & & $\mathrm{T} 144$ & puits $78 / 45$ & vers 11 avant J.-C. & tonneau incomplet & Sapin argenté & Noisetier \\
\hline & & T145 & puits $78 / 45$ & vers 11 avant J.-C. & tonneau incomplet & Sapin argenté & \\
\hline & & T146 & puits $78 / 45$ & vers 11 avant J.-C. & tonneau & Sapin argenté & \\
\hline & & $\mathrm{T} 147$ & puits 79/176 & vers 11 avant J.-C. & douelles & Sapin argenté & \\
\hline & & $\mathrm{T} 148$ & puits $79 / 176$ & vers 11 avant J.-C. & douelles & Sapin argenté & \\
\hline & & T149 & puits $82 / 29$ & vers 11 avant J.-C. & tonneau incomplet & Sapin argenté & \\
\hline & & $\mathrm{T} 150$ & puits $82 / 29$ & vers 11 avant J.-C. & tonneau & Sapin argenté & Noisetier \\
\hline & & T151 & puits $82 / 134$ & vers 11 avant J.-C. & tonneau incomplet & Sapin argenté & \\
\hline & & $\mathrm{T} 152$ & puits $82 / 134$ & vers 11 avant J.-C. & tonneau & Sapin argenté & Noisetier \\
\hline & & T153 & puits $82 / 214$ & vers 11 avant J.-C. & tonneau incomplet & Sapin argenté & \\
\hline
\end{tabular}




\begin{tabular}{|c|c|c|c|c|c|c|c|c|}
\hline ND & H & D & d & Capacité & $H / D$ & Marques & Restitution & Bibliographie \\
\hline & & & & & & C.S. AV+ & & Breuer, 1918 \\
\hline & & & & & & ONES-(..)I(...)NIT & & \\
\hline 17 & & & & & & M.DVN, ADVIV, C.C.P.L., S.S.D & & Bloemers, 1978 \\
\hline \multicolumn{9}{|l|}{18} \\
\hline 26 & $150 \mathrm{~cm}$ & $120 \mathrm{~cm}$ & $110 \mathrm{~cm}$ & 13601 & 1,25 & $\operatorname{CGM}(\ldots)$ & Vechten 107 & Ulbert, 1959 \\
\hline \multirow[t]{8}{*}{18} & & $75 \mathrm{~cm}$ & & & & & & \\
\hline & & & & & & & & \\
\hline & & & & & & & & Desbat, 1991 \\
\hline & & & & & & & & Ulbert, 1959 \\
\hline & & & & & & & & \\
\hline & & $86 \mathrm{~cm}$ & & & & & & Breuer, 1920 \\
\hline & & $86 \mathrm{~cm}$ & & & & & & \\
\hline & & & & & & $\mathrm{S}(\ldots) \mathrm{C}(\ldots), \mathrm{BFI}(\ldots)$ OU BII $(\ldots)$ & & \\
\hline 19 & & & $92 \mathrm{~cm}$ & & & & & Groeneveld, 1993 \\
\hline 18 & $197 \mathrm{~cm}$ & $104 \mathrm{~cm}$ & $80,5 \mathrm{~cm}$ & 10001 & 1,97 & S(...)PS, VIP. S, SVL“S, L. SVLP, I. ND, T. VC, CL. CC & Xanten 119 & \\
\hline \multicolumn{9}{|l|}{14} \\
\hline 19 & & & & 14401 & & LAC, TOS, SOT & & \\
\hline \multicolumn{9}{|l|}{14} \\
\hline \multirow[t]{11}{*}{18 à 20} & & & & & & & & Vollmer-König, 1990 \\
\hline & & & & & & & & Baratta, 1994 \\
\hline & & & & & & & & Kühlborn, 1992 \\
\hline & $150 \mathrm{~cm}$ & $74 \mathrm{~cm}$ & $57 \mathrm{~cm}$ & 4601 & 2,02 & & Oberaden 126 & \\
\hline & $196 \mathrm{~cm}$ & $95 \mathrm{~cm}$ & $80 \mathrm{~cm}$ & 10201 & 2,06 & & Oberaden 128 & \\
\hline & $205 \mathrm{~cm}$ & $95 \mathrm{~cm}$ & & & 2,15 & & Oberaden 129 & \\
\hline & $190 \mathrm{~cm}$ & $97 \mathrm{~cm}$ & $75 \mathrm{~cm}$ & 10201 & 1,95 & & Oberaden 131 & \\
\hline & $185 \mathrm{~cm}$ & $100 \mathrm{~cm}$ & $85 \mathrm{~cm}$ & $1280 I$ & 1,85 & & Oberaden 133 & \\
\hline & & $85 \mathrm{~cm}$ & & & & & & \\
\hline & & & & & & & & \\
\hline & & & & & & $\operatorname{SPAR}(\mathrm{I}), \operatorname{SOLVER}(\mathrm{I}), \mathrm{Q} . \operatorname{VETT}(\mathrm{I}),(\ldots) \operatorname{AP}(\mathrm{I})$ & & \\
\hline 20 & $155 \mathrm{~cm}$ & $78 \mathrm{~cm}$ & $68 \mathrm{~cm}$ & 5501 & 1,98 & & Oberaden 137 & \\
\hline \multicolumn{9}{|l|}{22} \\
\hline \multicolumn{9}{|l|}{22} \\
\hline \multirow[t]{2}{*}{38} & $200 \mathrm{~cm}$ & $90 \mathrm{~cm}$ & $70 \mathrm{~cm}$ & 9201 & 2,22 & & Oberaden 140 & \\
\hline & & $70 \mathrm{~cm}$ & & & & & & \\
\hline 31 & & $85 \mathrm{~cm}$ & & & & & & \\
\hline 39 & $190 \mathrm{~cm}$ & $85 \mathrm{~cm}$ & & & 2,23 & & Oberaden 143 & \\
\hline 30 & & & & & & T. VIREI, X & & \\
\hline 33 & & $90 \mathrm{~cm}$ & & & & GALLI, V(I)RATI & & \\
\hline \multirow[t]{5}{*}{27} & $210 \mathrm{~cm}$ & $103 \mathrm{~cm}$ & $85 \mathrm{~cm}$ & 13001 & 2,03 & $(\ldots) \mathrm{APA}(\ldots)$ & Oberaden 146 & \\
\hline & & & & & & & & \\
\hline & & $70 \mathrm{~cm}$ & & & & & & \\
\hline & $165 \mathrm{~cm}$ & $65 \mathrm{~cm}$ & & & 2,53 & & Oberaden 150 & \\
\hline & & $80 \mathrm{~cm}$ & & & & & & \\
\hline 22 & $165 \mathrm{~cm}$ & $73 \mathrm{~cm}$ & $60 \mathrm{~cm}$ & 5501 & 2,26 & SOLIVERI, T. VIREI & Oberaden 152 & \\
\hline & & & & & & & & \\
\hline
\end{tabular}


Tabl. V - Tableau récapitulatif des tonneaux antiques (suite).

\begin{tabular}{|c|c|c|c|c|c|c|c|}
\hline Région & Site & $\mathrm{N}^{\circ}$ & Contexte & Datation & Description & Essence(s) douelles & Essence cercles \\
\hline \multirow{34}{*}{$\begin{array}{l}\text { Limes } \\
\text { rhénan } \\
\text { (suite) }\end{array}$} & & T154 & puits $82 / 214$ & vers 11 avant J.-C. & tonneau avec 1 fond & Sapin argenté & \\
\hline & & T155 & puits $82 / 234$ & vers 11 avant J.-C. & tonneau incomplet & Sapin argenté & \\
\hline & & T156 & puits $82 / 234$ & vers 11 avant J.-C. & tonneau avcc 1 fond & Sapin argenté & Noisetier \\
\hline & & T157 & puits $83 / 1$ & vers 11 avant J.-C. & tonneau incomplet & Sapin argenté & Noisetier \\
\hline & & T158 & puits $84 / 64$ & vers 11 avant J.-C. & tonneau incomplet & Sapin argenté & \\
\hline & & T159 & puits $84 / 64$ & vers 11 avant J.-C. & tonneau incomplet & Sapin argenté & Noisetier \\
\hline & & T160 & puits $84 / 64$ & vers 11 avant J.-C. & tonneau incomplet & Sapin argenté & Noisetier \\
\hline & & T161 & puits $86 / 73$ & vers 11 avant J.-C. & tonneau incomplet & Sapin argenté & \\
\hline & & $\mathrm{T} 162$ & puits $86 / 73$ & vers 11 avant J.-C. & tonneau & Sapin argenté & Noisetier \\
\hline & Neuss & T163 & puits & & tonneau & & \\
\hline & \multirow[t]{2}{*}{ Saalburg } & T164 & puits & fin $I^{e r}$-début $\|^{e} s$. & tonneau & & \\
\hline & & T165 & & & deux douelles & conifère & \\
\hline & Okarben & T166 & & $\mathrm{f}^{\mathrm{er}} \mathrm{s}$. & & Sapin argenté & Hêtre \\
\hline & \multirow[t]{2}{*}{ Mayence } & T167 & puits & $\mathrm{fer}^{\mathrm{er}}$. & tonneau & & \\
\hline & & T168 & puits & fin $\|{ }^{e}$-début $1 I^{\mathrm{e}} s$. & tonncau & & \\
\hline & Gross Gerau & T169 & & & & & \\
\hline & Worms & $\mathrm{T} 170$ & puits & & huit douelles & & \\
\hline & Viernheim & $\mathrm{T} 171$ & & & & & \\
\hline & \multirow[t]{5}{*}{ Rheingonheim } & $\mathrm{T} 172$ & puits & Ier $s$ & tonneau incomplet & Sapin & \\
\hline & & $\mathrm{T} 173$ & puits & & tonneau & & \\
\hline & & $\mathrm{T} 174$ & puits & $\mathrm{e}^{\mathrm{er}} \mathrm{s}$ & tonneau & & \\
\hline & & $\mathrm{T} 175$ & puits & $\mathrm{fer}^{\mathrm{er}}$ & tonneau & & \\
\hline & & T176 & puits & $\mathrm{fer}^{\mathrm{er}} \mathrm{s}$ & tonneau incomplet & & \\
\hline & Rheinzabern & $\mathrm{T} 177$ & & & deux douelles & conifère & \\
\hline & \multirow[t]{9}{*}{ Strasbourg } & $\mathrm{T} 178$ & puits & début $\mathrm{l}^{\mathrm{er}} \mathrm{s}$. & tonneau incomplet & Châtaigner & \\
\hline & & T179 & puits & $1^{\text {re }}$ moitié $\mathrm{J}^{\mathrm{er}} \mathrm{s}$. & tonneau & Sapin & \\
\hline & & T180 & puits & $\mathrm{f}^{\mathrm{er}} \mathrm{s}$ & tonneau & Hêtre & \\
\hline & & T181 & puits & fer $s$ & tonneau & & \\
\hline & & T182 & puits & vers 100 de notre ère & tonneau & & \\
\hline & & T183 & puits & III s. & tonneau & & \\
\hline & & T184 & puits & avant fin $\mathrm{II}^{\mathrm{e}} \mathrm{s}$. & tonneau incomplet & & \\
\hline & & T185 & puits & & tonneau incomplet & Chêne & \\
\hline & & T186 & puits & & tonneau & & \\
\hline & Ohrigen & T187 & & & & & \\
\hline \multirow{14}{*}{$\begin{array}{l}\text { Limes } \\
\text { danubien }\end{array}$} & Risstissen & T188 & puits & & & & \\
\hline & Kempten & $\mathrm{T} 189$ & & & & & \\
\hline & Augsburg & T190 & & & & & \\
\hline & \multirow[t]{2}{*}{ Regensburg } & T191 & puits & $70-100$ & six douelles & Sapin & Hêtre \\
\hline & & T192 & puits & $70-100$ & tonneau incomplct & Sapin & Hêtre \\
\hline & Manching & T193 & fosse & La Tène finale & tonneau carbonisé & Sapin & \\
\hline & Carnuntum & T194 & puits & avant milieu $\mathrm{I}^{\mathrm{er}} \mathrm{s}$. & tonneau incomplet & & \\
\hline & \multirow[t]{7}{*}{ Budapest } & T195 & puits & avant milieu $\|^{\mathrm{e}} \mathrm{s}$. & tonneau incomplet & Pin & \\
\hline & & T196 & puits & avant milieu $\|^{\mathrm{e}} \mathrm{s}$. & tonneau incomplet & Chêne & Peuplier \\
\hline & & T197 & puits & avant milieu $\|^{\mathrm{e}} \mathrm{s}$. & tonneau avec bouchon & & \\
\hline & & T198 & puits & & douelles & & \\
\hline & & T199 & puits & & douelle avec bonde & & \\
\hline & & T200 & puits & & douelle & & \\
\hline & & T201 & puits & & douelle & & \\
\hline Italie & Grado & T202 & épave & fin $\|^{\mathrm{e}}$-début $\|^{\mathrm{e}} \mathrm{s}$. & tonneau & & \\
\hline
\end{tabular}




\begin{tabular}{|c|c|c|c|c|c|c|c|c|}
\hline ND & H & D & d & Capacité & H/D & Marques & Restitution & Bibliographie \\
\hline \multirow[t]{3}{*}{28} & $163 \mathrm{~cm}$ & $70 \mathrm{~cm}$ & $60 \mathrm{~cm}$ & 4801 & 2,32 & SIIGIATI, L. EPIDI, .CR, XXX, XC & Oberaden 154 & \\
\hline & & & & & & & & \\
\hline & $150 \mathrm{~cm}$ & $78 \mathrm{~cm}$ & & & 1,92 & & Oberaden 156 & \\
\hline 35 & & & & & & & & Kühlborn, 1992 \\
\hline \multicolumn{9}{|l|}{26} \\
\hline \multicolumn{9}{|l|}{36} \\
\hline \multicolumn{9}{|l|}{27} \\
\hline & & & & & & & & \\
\hline \multirow[t]{2}{*}{28} & $105 \mathrm{~cm}$ & $75 \mathrm{~cm}$ & & & 1,4 & & Oberaden 162 & \\
\hline & & & & & & & & Ulbert, 1959 \\
\hline \multirow[t]{8}{*}{21} & $216 \mathrm{~cm}$ & & & & & & & Ulbert, 1959 \\
\hline & & & & & & SENTIOR, VM & & Breuer, 1920 \\
\hline & & & & & & & & Hopf, 1980 \\
\hline & $178 \mathrm{~cm}$ & $90 \mathrm{~cm}$ & $75 \mathrm{~cm}$ & 8801 & 1,98 & & Mayence 167 & Ulbert, 1959 \\
\hline & $205 \mathrm{~cm}$ & & & & & & & \\
\hline & & & & & & & & Baratta, 1994 \\
\hline & & & & & & & & Ulbert, 1959 \\
\hline & & & & & & & & Baratta, 1994 \\
\hline 16 & & & & & & L. E. S., TIV. SVC, DO. SVC & & Ulbert, 1959 \\
\hline \multirow[t]{5}{*}{20} & & & & & & PERPETVI, PIGILLI, COBNERTI, VENVSTI, R. S & & \\
\hline & & & & & & & & \\
\hline & & & & & & & & \\
\hline & & & & & & & & \\
\hline & & & & & & SENTIOR & & Ulbert, 1959 \\
\hline \multirow[t]{2}{*}{$19-20$} & & & & & & & & Audin, 1985 \\
\hline & $180 \mathrm{~cm}$ & $86 \mathrm{~cm}$ & $68 \mathrm{~cm}$ & 7501 & 2,09 & & Strasbourg 179 & Toutain, 1920 \\
\hline \multirow[t]{12}{*}{18} & $194 \mathrm{~cm}$ & $100 \mathrm{~cm}$ & $80 \mathrm{~cm}$ & 11001 & 1,94 & & Strasbourg 180 & \\
\hline & & & & & & & & Ulbert, 1959 \\
\hline & & & & & & & & \\
\hline & & & & & & & & \\
\hline & & & $66 \mathrm{~cm}$ & & & & & Hatt, 1970 \\
\hline & & & & & & & & Ulbert, 1959 \\
\hline & & & & & & & & \\
\hline & & & & & & & & Baratta, 1994 \\
\hline & & & & & & RIPA & & Ulbert, 1959 \\
\hline & & & & & & & & Baratta, 1994 \\
\hline & & & & & & & & Baratta, 1994 \\
\hline & & & & & & & & Ulbert, 1959 \\
\hline \multirow[t]{3}{*}{22} & $160 \mathrm{~cm}$ & $80 \mathrm{~cm}$ & $64 \mathrm{~cm}$ & 5601 & 2 & L. I. MATV, P. L. A, CAVI. ALIOS COM(...,/ORCASTO(...) & Regensburg 192 & \\
\hline & & & & & & & & Baratta, 1994 \\
\hline & & $58 \mathrm{~cm}$ & & & & & & Swoboda, 1958 \\
\hline 21 & & $76 \mathrm{~cm}$ & & & & IMMVNE IRN VAL LEG II AD & & Bezeczky, 1996 \\
\hline 22 & $154 \mathrm{~cm}$ & & & & & MCO (...) MCATR, R XXIIII & & Ulbert, 1959 \\
\hline \multirow[t]{6}{*}{22} & $158 \mathrm{~cm}$ & $85 \mathrm{~cm}$ & $63 \mathrm{~cm}$ & 6201 & 1,85 & Illisible & Budapest 197 & \\
\hline & & & & & & EXPAC NTR VAL LEG II ADI & & Bezeczky, 1996 \\
\hline & & & & & & $M(\ldots) V I L /(\ldots) R I$ & & \\
\hline & & & & & & $\mathrm{IM}(\ldots)$ & & Ulbert, 1959 \\
\hline & & & & & & IMMVNE IRN VAL LEG II AD & & \\
\hline & & & & & & & & Dell'Amico et al., 1991 \\
\hline
\end{tabular}

